OPEN ACCESS

Edited by:

Weilin Xu,

Zhejiang University, China

Reviewed by:

Zhijun Zhang,

Shanghai Jiao Tong University, China

Antonio Vinciguerra,

University of Naples Federico II, Italy

${ }^{*}$ Correspondence:

Qichuan Zhuge

qc.zhuge@wmu.edu.cn

Su Yang

yangsu@wmu.edu.cn;

yangsu0575@163.com

Specialty section:

This article was submitted to

Neurodegeneration,

a section of the journal

Frontiers in Neuroscience

Received: 09 June 2020

Accepted: 21 July 2020

Published: 18 August 2020

Citation:

Wang $K$, Ru J, Zhang $H$, Chen J,

Lin X, Lin Z, Wen M, Huang L, Ni H, Zhuge $Q$ and Yang S (2020) Melatonin

Enhances the Therapeutic Effect of Plasma Exosomes Against Cerebral Ischemia-Induced Pyroptosis Through

the TLR4/NF- $\kappa B$ Pathway.

Front. Neurosci. 14:848.

doi: 10.3389/fnins.2020.00848

\title{
Melatonin Enhances the Therapeutic Effect of Plasma Exosomes Against Cerebral Ischemia-Induced Pyroptosis Through the TLR4/NF-кB Pathway
}

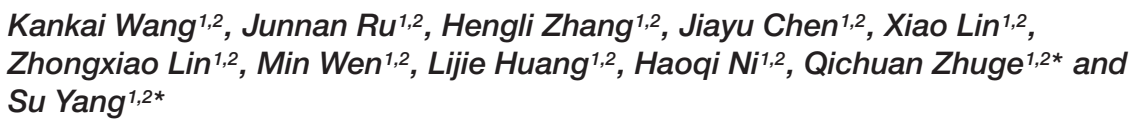

${ }^{1}$ Zhejiang Provincial Key Laboratory of Aging and Neurological Disorder Research, The First Affiliated Hospital of Wenzhou Medical University, Wenzhou, China, ${ }^{2}$ Department of Neurosurgery, The First Affiliated Hospital of Wenzhou Medical University, Wenzhou, China

Introduction: Ischemic stroke-induced inflammation and inflammasome-dependent pyroptotic neural death cause serious neurological injury. Nano-sized plasma exosomes have exhibited therapeutic potential against ischemia and reperfusion injury by ameliorating inflammation. To enhance its therapeutic potential in patients with ischemic injury, we isolated exosomes from melatonin-treated rat plasma and assessed the neurological protective effect in a rat model of focal cerebral ischemia.

Methods: Basal plasma exosomes and melatonin-treated plasma exosomes were isolated and intravenously injected into a rat model of focal cerebral ischemia. Neurological recovery was evaluated by determining the modified neurological severity score (mNSS), infarct volume, and brain water content. Pyroptosis in the ischemic cortex was detected through dUTP nick-end labeling (TUNEL) assay, lactate dehydrogenase $(\mathrm{LDH})$ release, and gasdermin D (GSDMD) cleavage. NLRP3 inflammasome assembly and global inflammatory cytokine secretion were detected by enzyme-linked immunosorbent assay (ELISA) and Western blot assay. In immunized Sprague-Dawley rats, microglia pyroptosis was determined through a positive percentage of $\mathrm{IBA}^{+}$and caspase-1 (p20) ${ }^{+}$cells. Finally, the microRNA (miRNA) profiles in melatonin-treated plasma exosomes were analyzed by exosome miRNA microarray analysis.

Results: Melatonin treatment enhanced plasma exosome therapeutic effects against ischemia-induced inflammatory responses and inflammasome-mediated pyroptosis. In addition, we confirmed that ischemic stroke-induced pyroptotic cell death occurred in the microglia and neuron, while the administration of melatonin-treated exosomes further effectively decreased the infarct volume and improved recovery of function via regulation of the TLR4/NF-kB signaling pathway. Finally, the altered miRNA profiles in the melatonin-treated plasma exosomes demonstrated the regulatory mechanisms involved in neurological recovery after ischemic injury. 
Conclusion: This study suggests that nano-sized plasma exosomes with melatonin pretreatment might be a more effective strategy for patients with ischemic brain injury. Further exploration of key molecules in the plasma exosome may provide increased therapeutic value for cerebral ischemic injury.

Keywords: ischemic stroke, melatonin, plasma exosome, pyroptosis, inflammatory response, TLR4/NF-кB signaling pathway

\section{INTRODUCTION}

According to the Global Burden of Disease Study, stroke has become the second leading cause of death and the third leading cause of global disability-adjusted life years, putting tremendous pressure on medical care worldwide (Feigin et al., 2014). As a sudden neurological dysfunction caused by interruption of the blood supply, various pharmacological and cytological therapies have been applied to mitigate post-stroke injury and improve recovery of neurological function. Growing evidence suggests that the inflammatory response and inflammatory cell death play crucial roles in the secondary injury in ischemic stroke (Jin et al., 2010). Although a vast number of systemic studies have been devoted to elucidating the internal mechanisms, there is still missing information and the mechanism content is constantly updated.

Pyroptosis was identified as a programmed form of necrosis, which features a lytic process featuring cell swelling and large bubbles emanating from the plasma membrane (Sarhan et al., 2018). As a high level of inflammatory response, pyroptosis is triggered by nucleotide-binding oligomerization domain-like receptors (NLRs), which recognize various pathological stimuli, including pathogen-associated molecular patterns (PAMPs) and endogenous damage-associated molecular patterns (DAMPs). Important compounds of the inflammasome are the NLRPs (NOD-like receptors containing pyrin domains), which consist mainly of NLRP3 and NLRP1. NLRP3 interacts with procaspase- 1 upon connection with the apoptosis-associated specklike protein containing the CARD (ASC) adapter, leading to the cleavage of precursors of inflammatory cytokines into mature forms, including interleukin-18 (IL-18) and interleukin$1 \beta$ (IL-1 $\beta$ ) (He et al., 2016). Activated caspase-1 subsequently cleaves and releases the $\mathrm{N}$-domain of gasdermin D (GSDMD), which eventually forms membrane pores and releases mature inflammatory mediators into the extracellular space, causing a severe inflammatory cascade reaction (Liang and Liu, 2016). Studies have shown that inflammation-related pyroptosis is an important factor affecting stroke prognosis (Jin et al., 2010). In a rat stroke model, the protein GSDMD promptly increased and then climbed to a peak $24-48 \mathrm{~h}$ post-ischemia, indicating that pyroptosis occurs efficiently during cerebral ischemia injury (Zhang D. et al., 2019).

Exosomes are nano-sized extracellular vesicles (EVs) secreted by living cells into the extracellular fluids, and biofluids such as blood, urine, and cerebrospinal fluid have all been proven to secrete exosomes (Zhang et al., 2015). Recently, exosomes have received widespread attention for their inclusive composition (Zhang X. et al., 2019). These nanocarriers contain proteins, lipids, and nucleic acids, which are the major mediators of cell-cell communication and play regulatory roles in several cellular processes such as immune regulation (Vanni et al., 2017; Zhang X. et al., 2019). In addition, plasma exosomes from young individuals have been reported to have antiinflammatory effects on ischemia (Lee B.R. et al., 2018); nevertheless, the mechanism by which exosomes protect nerve cells from ischemia injury remains unclear. Numerous studies have confirmed that the microRNAs (miRNAs) in exosomes function as key factors in the regulatory process of inflammation, angiogenesis, cellular transport, apoptosis, and proteolysis (Eirin et al., 2014; Liu et al., 2016). A class of exosomal miRNAs such as microRNA-29a, microRNA-9, microRNA-30c-5p, microRNA$30 \mathrm{~d}$, and microRNA-155 have been proven to modulate the cell pyroptotic process after brain injury (Li et al., 2014; Jeyabal et al., 2016; Wu et al., 2016; Minghua et al., 2018; Ding et al., 2020). Previous studies have shown altered miRNA profiles in the plasma or exosomes in various physiological environments or after pharmacological treatments (Minghua et al., 2018; Yoon et al., 2020; Zhang and Jin, 2020). Therefore, it is of significant interest to determine the composition and function of exosomal miRNAs in inflammasome-dependent pyroptosis during cerebral ischemic injury.

Melatonin is a potent free radical scavenger and broadspectrum antioxidant that is synthesized by the pineal gland or other organs and has been shown to inhibit inflammation and apoptosis in cerebral ischemic injury (Rancan et al., 2018). However, the specific mechanism has not yet been clearly explained. Interestingly, some studies have found that melatonin can affect the composition of exosomes, such as microRNA (Cheng et al., 2017; Alzahrani, 2019; Hunsaker et al., 2019; Yoon et al., 2020), suggesting that it has versatile therapeutic means to improve stroke prognosis. In this study, we investigated the therapeutic effect of plasma exosomes against ischemia-induced inflammation and inflammasome-dependent pyroptosis. Based on these findings, we suggest that melatonin alters the microRNA profiles in plasma exosomes and ultimately improves stroke recovery. However, the correlation between the anti-pyroptotic mechanisms and the specific miRNA functions requires further research.

\section{MATERIALS AND METHODS}

\section{Animals and Ethics Statement}

Sprague-Dawley rats weighing 200-250 g were purchased from the Shanghai Charles River Experimental Animal Limited Liability Company (Shanghai, China). The animals were 
housed in a standardized animal care center with appropriate temperature and humidity with a $12 \mathrm{~h}$ light/dark cycle. Rats were given free access to food and water. All experimental procedures were approved by the Ethics Committee of Wenzhou Medical University and were carried out in strict accordance with the animal care and use guidelines of the National Institutes of Health.

\section{Stroke Model and Exosome Injection}

The permanent distal middle cerebral artery occlusion (pMCAO) model was used to create permanent focal ischemia, as previously described (Ren et al., 2018). Briefly, Sprague-Dawley rats were anesthetized and incised between their left eye socket and tragus. With the temporal muscles separated, a $3 \mathrm{~mm}$ diameter bone window was drilled for left middle cerebral artery exposure. After separation and blocking of the common carotid arteries on both sides, the distal middle cerebral artery was cauterized for permanent blocking. The carotid arteries were then released, and wounds were sutured with continual vital signs monitoring. The sham group underwent the same surgical procedure without cerebral artery blocking. To explore the influence of exosomes (Exo) and melatonin-Exo on pMCAO-induced cerebral injury, exosomes derived from rat plasma suspended in phosphatebuffered saline (PBS) were injected intravenously through the tail vein at a concentration of $100 \mu \mathrm{g}$ per rat at 1,12 , and $36 \mathrm{~h}$ after cerebral artery blocking. The pMCAO group received an equal volume of PBS. All experiments followed the schedule shown in Figure 1A.

\section{Isolation and Characterization of Serum Exosomes}

Melatonin dissolved in $0.9 \%$ saline (with 5\% DMSO) was injected intraperitoneally once a day for seven consecutive days $(10 \mathrm{mg} / \mathrm{kg})$. At day 8 , the rats were sacrificed and blood was collected for plasma acquisition. Plasma exosomes were then extracted with a Total Exosome Isolation Kit (Invitrogen). The isolated exosomes were suspended in PBS for further characterization. Exosome size distribution and concentration were analyzed using a ZETASIZER Nano series-Nano-ZS system. Vesicles were visualized by light scattering using a Hitachi $\mathrm{H}-7650$ transmission electron microscope. Specific exosome markers including TSG101, CD63, and cytochrome C were determined through Western blot analysis.

\section{TTC Staining}

Three days after stroke, the brain was excised and cut into five pieces. The sections were then immersed in preheated 2\% 2,3,5-triphenyltetrazolium chloride (TTC; Sigma Aldrich) solution for $20 \mathrm{~min}$. After washing with PBS, the sections were placed with the frontal pole toward the front and captured with a camera. Finally, the infarct volume was calculated by ImageJ software.

\section{Neurobehavioral Training and Evaluation}

The modified neurological severity score (mNSS) test was used to assess neurological deficits, as described previously (Longa et al., 1989). The mNSS includes multiple tasks to assess motor,

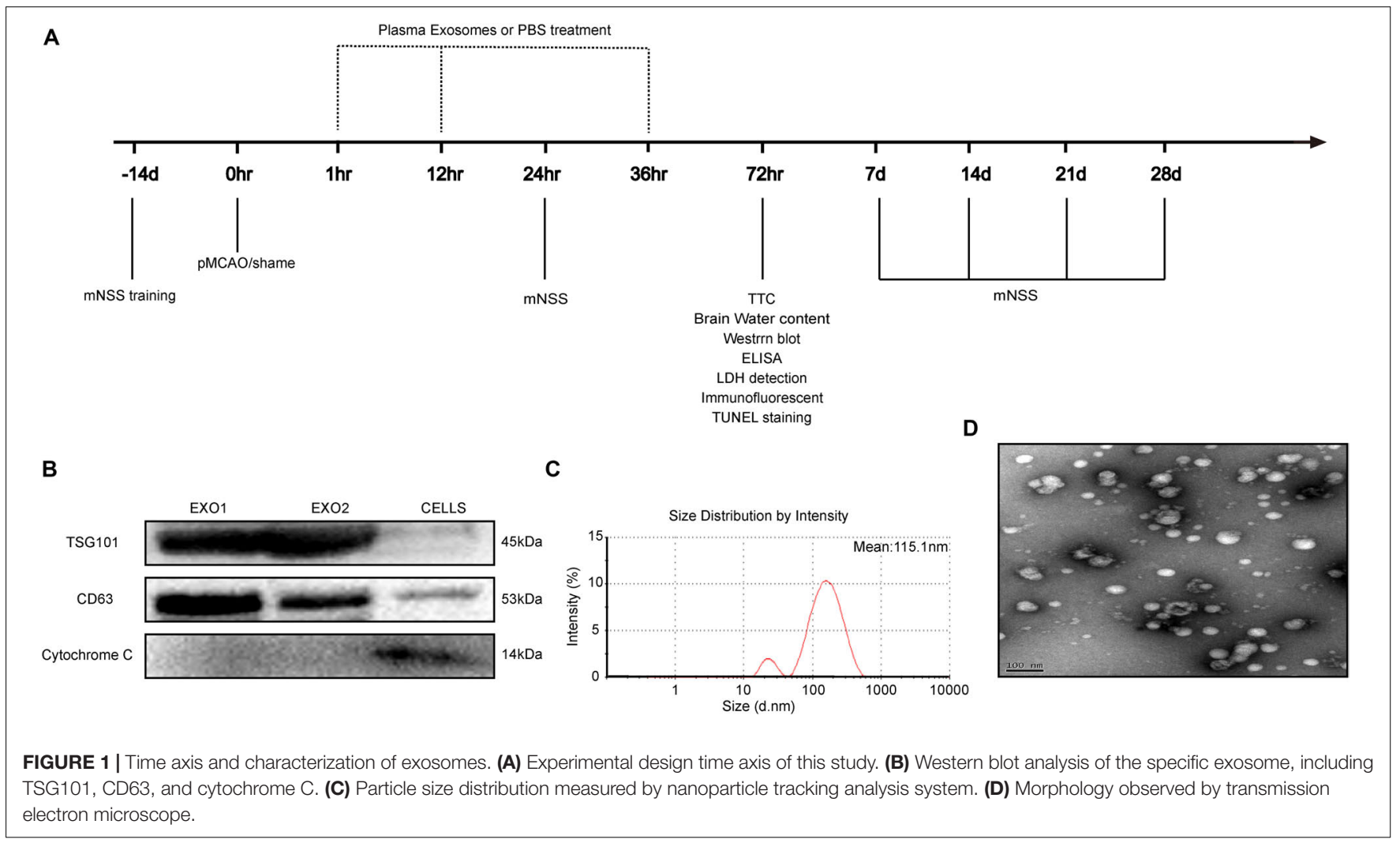


sensory, reflex, and balance abilities and is conducted at 1, 7, 14, 21 , and 28 days after surgery, with a maximum deficit score of 18 . A higher score indicates more severe neurological dysfunction. All mice were pre-trained for 14 days before the operation until their performance reached a steady state.

\section{Assessment of Cerebral Edema}

Brain water content (BWC) was evaluated as previously described (Ishrat et al., 2015). Rat brain tissues were taken 3 days after surgery, and the ipsilateral and contralateral sides were then separated and weighed for wet weight (WW). The tissues were dried in an oven at $100^{\circ} \mathrm{C}$ for $24 \mathrm{~h}$ to obtain a dry weight (DW). BWC was calculated using the following formula: $100 \% \times(\mathrm{WW}-\mathrm{DW}) / \mathrm{WW}$.

\section{Western Blot Analysis}

Total proteins were extracted from brain tissues using RIPA lysis buffer (Thermo Fisher Scientific, United States) and quantified with a bicinchoninic acid (BCA) Protein Assay Kit (Thermo Fisher Scientific, United States) according to the manufacturer's instructions. Sodium dodecyl sulfate polyacrylamide gel electrophoresis (SDS-PAGE) was then conducted. After being transferred onto polyvinylidene fluoride (PVDF) membranes, the membranes were blocked with $5 \%$ milk for $2 \mathrm{~h}$, followed by incubation at $4^{\circ} \mathrm{C}$ for $24 \mathrm{~h}$ with primary antibodies against the following proteins: $\alpha$-tubulin (1:1,000, CST, Shanghai, China); NLRP3 (1:1,000, CST, Shanghai, China); ASC (1:1,000, Santa Cruz, Shanghai, China); caspase-1 (1:1,000, CST, Shanghai, China); GSDMD (1:1,000, Abcam, Cambridge, United Kingdom); TLR4 (1:1,000, Abcam, Cambridge, United Kingdom); and NF- $\mathrm{B}$ (1:1,000, Abcam, Cambridge, United Kingdom). The membrane was further incubated with a secondary antibody and then detected using the Bio-Rad ChemiDoc XRS imaging system. ImageJ software was used to analyze immunoreactive bands. The target protein signal intensities were compared to either the $\alpha$-tubulin or $\beta$-actin intensity.

\section{Enzyme-Linked Immunosorbent Assay and Serum LDH Detection}

The secretion levels of TGF- $\beta$, TNF- $\alpha$, IL-6, IL-1 $\beta$, IL-10, IL-18, TLR4, HMGB1, and NF-кB were measured using an enzymelinked immunosorbent assay (ELISA) kit (Beyotime, Shanghai, China) according to the manufacturer's instructions. Plasma lactate dehydrogenase (LDH) detection was measured using an LDH activity detection kit (Solarbio, Beijing, China).

\section{Immunofluorescence}

Brain sections were fixed with $4 \%$ paraformaldehyde at room temperature for $20 \mathrm{~min}$ and then incubated in PBST $(0.4 \%$ triton in PBS) containing 5\% bovine serum albumin (BSA) solution (Santa Cruz, Shanghai, China) for 30 min to block nonspecific staining. Next, the sections were incubated with primary antibodies at $4^{\circ} \mathrm{C}$ overnight, followed by incubation with the corresponding secondary antibody at $37^{\circ} \mathrm{C}$ for $1 \mathrm{~h}$. The nuclei were counterstained with DAPI (Abcam). Then, the sections were examined with a scanning fluorescence microscope (Leica Microsystems) and analyzed using ImageJ software.

\section{TdT-Mediated dUTP Nick-End Labeling Assays}

A One Step dUTP nick-end labeling (TUNEL) Apoptosis Assay Kit (Beyotime, Shanghai, China) was used to detect cell apoptosis according to the manufacturer's instructions. Briefly, the cells were incubated with $50 \mu$ l TUNEL detection mixture at $37^{\circ} \mathrm{C}$ for $1 \mathrm{~h}$ in the dark and rinsed in PBS. Subsequently, $50 \mu l$ streptavidin-horseradish peroxidase (HRP) solution was added and incubated for $30 \mathrm{~min}$ at room temperature. DAB staining was then performed, followed by hematoxylin staining of the nuclei.

\section{miRNA Microarray Analysis}

The NEB Next Multiplex Small RNA Library Prep Set for Illumina (NEB, United States) was used for RNA library preparation according to the manufacturer's protocol. Briefly, total RNA extracted from each plasma exosome sample was ligated with $3^{\prime}$ and $5^{\prime}$ adapters for Illumina. After sample quality control and quantification with a NanoDrop ND-1000, reverse transcription was conducted using ProtoScript II Reverse Transcriptase (NEB, United States). An Agilent 2100 Bioanalyzer was used for library quality control and quantification. The cDNA library for 135-155 bp (corresponding to single-end small RNA at 15-35 nt) was selected for miRNA sequencing with an Illumina NextSeq 500 instrument (Illumina Inc., United States). Solexa CHASTITY was used for quality screening of clean reads, and the $3^{\prime}$ ends of the sequenced reads were removed to obtain trimmed reads that were at least 15 nucleotides in length. Filtered reads were annotated using miRDeep2. Altered levels of the miRNA expressions (normalized) in exosomes were calculated with fold change $>1.5$ and $P$-values $<0.05$ (Student's $t$-test).

\section{Target Prediction and Bioinformatics Analysis}

The sequencing data have been deposited in the NCBI Gene Expression Omnibus $\left(\mathrm{GEO}^{1}\right)$ with the accession number GSE147578.

To detect the top expressed miRNAs in plasma exosomes and alternative expressions after melatonin treatment, edgeR was used to identify differentially expressed genes. All predicted genes with a target prediction score $\geq 80$ were subjected to Gene Ontology (GO) for gene enrichment and functional annotation analyses.

\section{Statistical Analysis}

GraphPad Prism software was used for the statistical analyses. The measured data are expressed as the mean \pm SD. Differences between the two groups were evaluated by Student's $t$-test. Analysis of variance (ANOVA) was used to compare three or more groups. $P<0.05$ was considered statistically significant.

\footnotetext{
${ }^{1}$ http://www.ncbi.nlm.nih.gov/geo/
} 


\section{RESULTS}

\section{Isolation and Identification of Exosomes}

Adult male rats (200-250 g) received intraperitoneal injections for seven consecutive days with a control solvent (5\% DMSO + PBS) or melatonin (10 mg/kg, dissolved in $5 \%$ DMSO + PBS). On day 8, plasma exosomes (EXO1 represented basic plasma exosomes and $\mathrm{EXO} 2$ represented melatonin-treated plasma exosomes) were isolated and identified. Concurrently, cell extraction from the microglial BV2 cell line was selected as a control for exosome identification. We detected the characteristic biomarkers of exosomes, TSG101 and CD63, which were expressed at higher levels in the exosomes than in the control cells, while cytochrome C (negative marker for exosomes) was absent or underrepresented in the exosomes (Figure 1B). The spherical morphology characteristics and a diameter of 30$150 \mathrm{~nm}$ with an average particle size of $115.1 \mathrm{~nm}$ were confirmed through nanoparticle tracking analysis (NTA) (Figure 1C) and transmission electron microscope (TEM) (Figure 1D) and were in agreement with other studies (Lotvall et al., 2014). Taken together, these data demonstrate that the isolates had all the characteristics of purified exosomes.

\section{Melatonin Enhances the Therapeutic Effect of Exosomes by Reducing Brain Damage and Promoting Functional Recovery After Stroke}

Studies have confirmed the therapeutic effect of young rat plasma exosomes on cardiac ischemia-reperfusion injury and angiogenesis (Vicencio et al., 2015; Chen et al., 2020). To investigate the effect of melatonin on exosomes in the treatment of ischemic stroke, plasma exosomes from melatonin- or PBStreated adult rats were prepared for treatment. As shown in Figure 2, the EXO1 group (pMCAO with EXO1 treatment, $100 \mu \mathrm{g}$ ) and the EXO2 group (pMCAO with EXO2 treatment, $100 \mu \mathrm{g}$ ) had lower mNSS than the pMCAO group (equal volume of PBS treatment). At 28 days after stroke, the EXO1 group score was $5.25 \pm 0.4523$, which was significantly lower than that of the PMCAO group, approximately $6.583 \pm 0.5149$

\section{A}

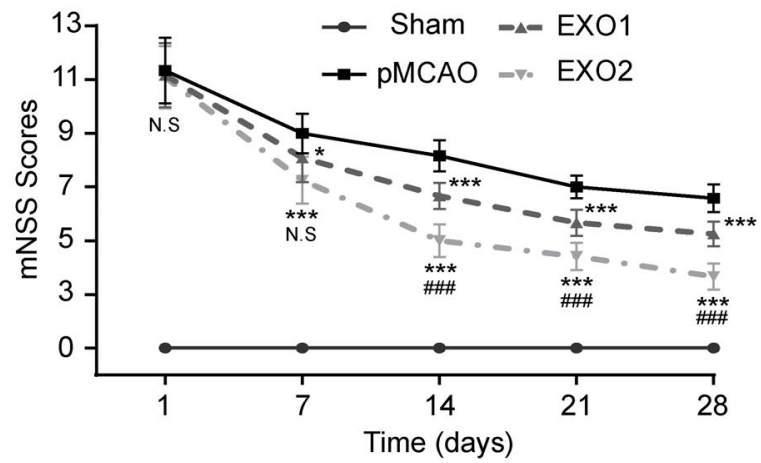

C

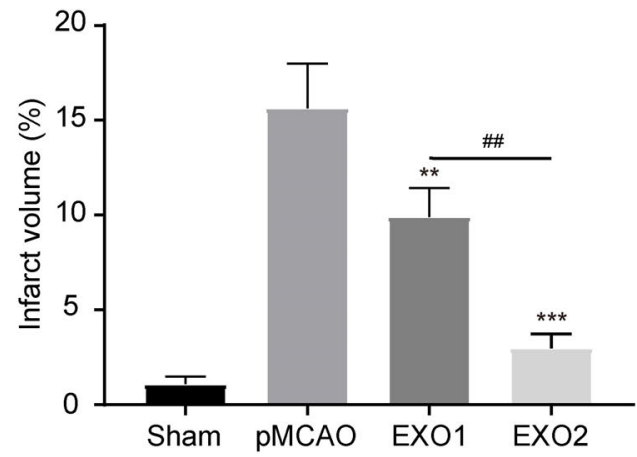

B

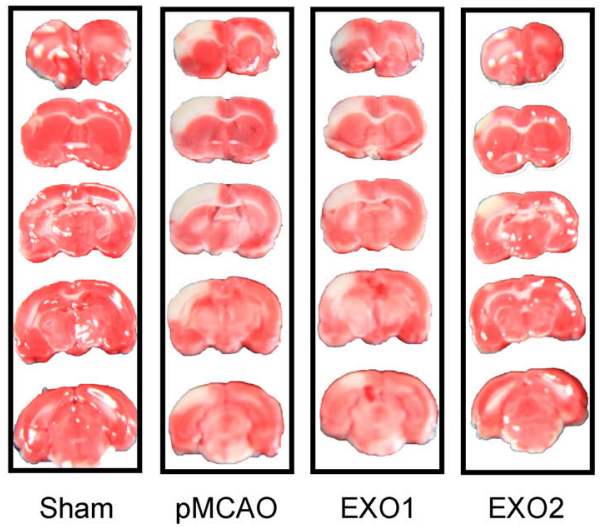

D

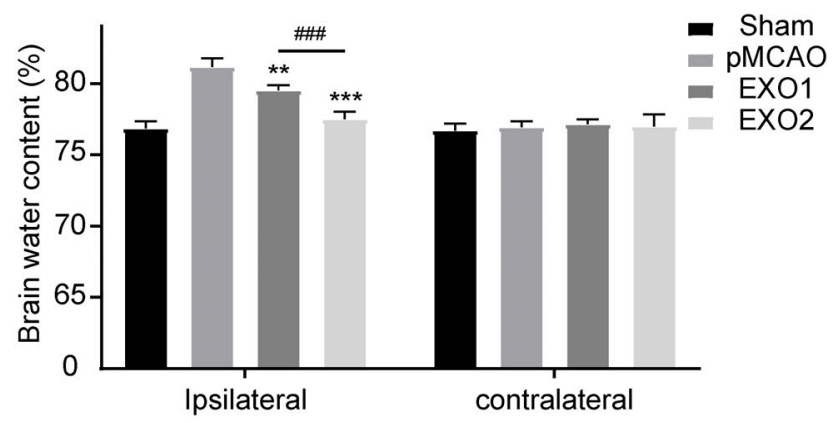

FIGURE 2 | Exosome treatment reduced brain damage and promoted functional recovery after stroke. (A) Neurological recovery was evaluated by the modified neurological severity score (mNSS) at 1, 7, 14, 21, and 28 days post-permanent distal middle cerebral artery occlusion (pMCAO, $n=12$ ). (B,C) Representative images of 2,3,5-triphenyltetrazolium chloride (TTC) staining in five sequential brain slices from the various groups of rats $(n=3)$. (D) Brain water content was evaluated at $72 \mathrm{~h}$ post-injury $(n=4)$. Data are presented as the mean $\pm \mathrm{SD}$. ${ }^{\star} P<0.05,{ }^{\star \star} P<0.01,{ }^{\star \star \star} P<0.001$ vs. the pMCAO group. ${ }^{\# \#} P<0.01$, ${ }^{\# \# \#} P<0.001$ vs. the EXO1 (basic plasma exosomes) group. 
$(P<0.001)$. Whereas the score of the EXO2 group was even lower at $3.667 \pm 0.4924$, which was more significant than that of the EXO1 group $(P<0.001)$, indicating that the EXO2 group received better therapeutic action. Similarly, the TTC staining assay showed that the cerebral infarction of the EXO1 group exhibited a smaller infarct size of $9.915 \pm 1.504 \%$ compared to $15.64 \pm 2.354 \%$ in the pMCAO group $(P<0.05)$. The infarct size decreased even further to $2.98 \pm 0.7406 \%$ in the EXO2 group $(P<0.01$, compared with EXO1), suggesting that melatonin enhanced the effect of exosomes on reducing the infarct volume. Moreover, the BWC assay showed that the brain water content of the EXO1 and EXO2 groups decreased to $79.525 \pm 0.358 \%(P<0.01$, compared with the pMCAO group) and $77.505 \pm 0.527 \%$ ( $P<0.001$, compared with the EXO1 group), respectively, compared with the pMCAO group $(81.178 \pm 0.584)$ and sham group (76.840 \pm 0.515$)$, suggesting that EXO2 is more significant for the prognosis of stroke in rats. Therefore, we believe that melatonin enhanced the plasma exosomes' ability to improve neural functional recovery.

A
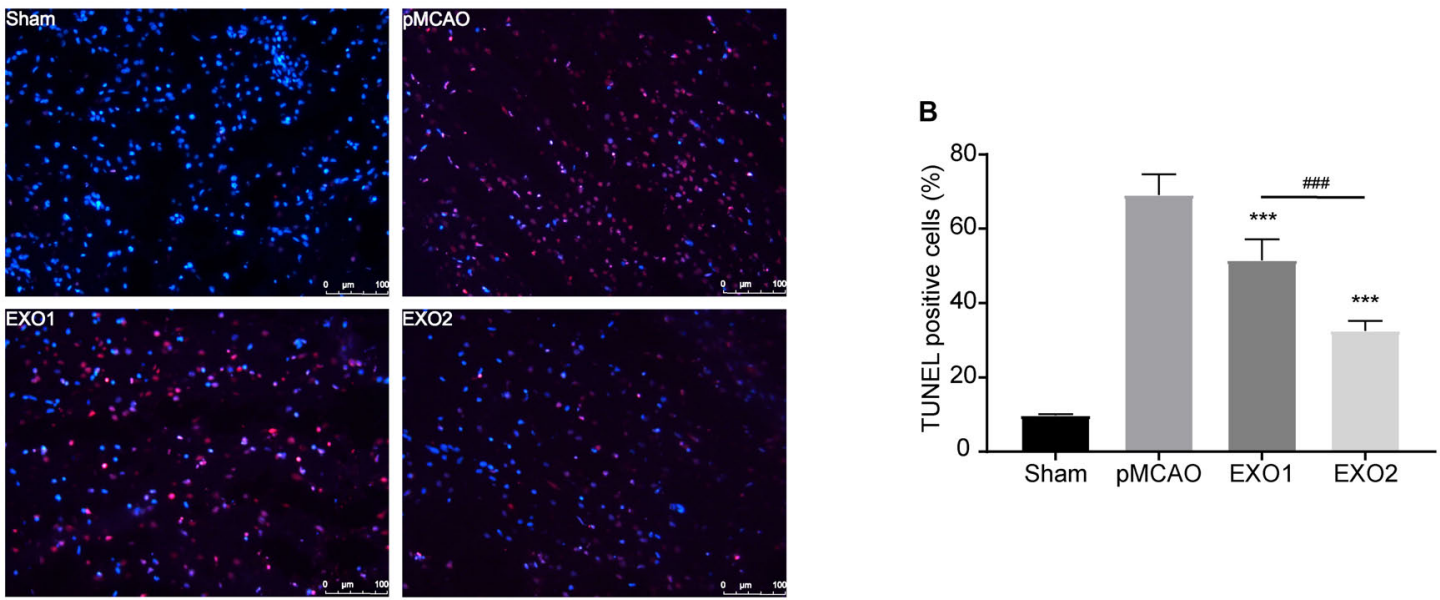

C

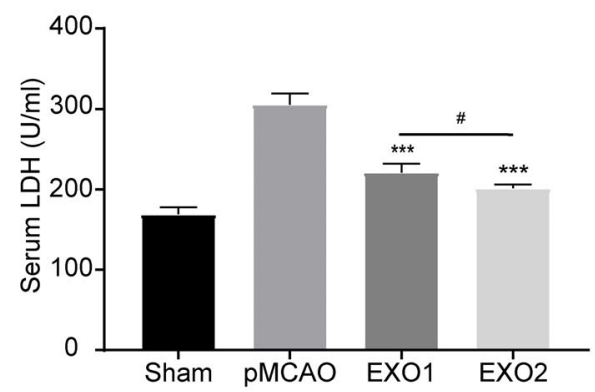

D

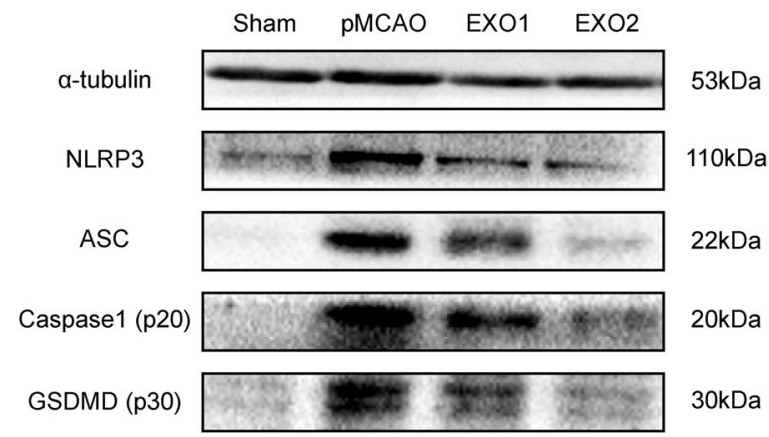

E

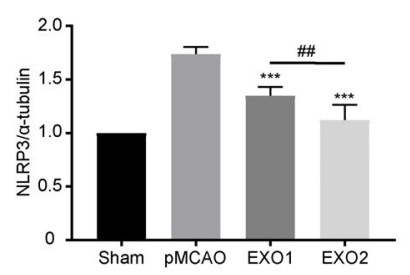

$\mathbf{F}$

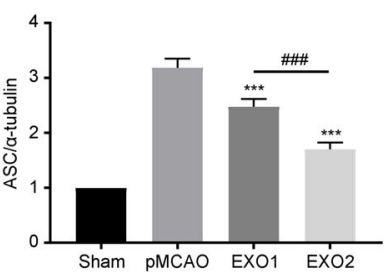

G

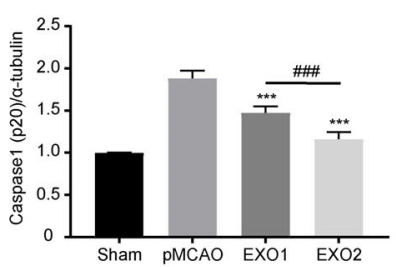

H

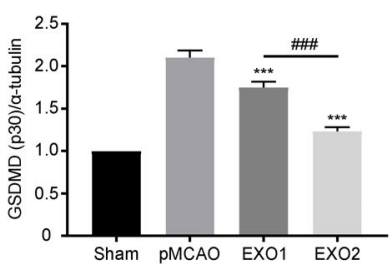

FIGURE 3 | Administration of basic plasma exosomes (EXO1) and melatonin-treated plasma exosomes (EXO2) inhibited NLRP3-dependent pyroptosis in the ischemic cortex. (A) dUTP nick-end labeling (TUNEL) assay was used to detect the apoptosis of cortical nerve cells post-ischemic injury. Representative images of the TUNEL-positive apoptotic cells (red) in sagittal brain sections at day 3 post-injury. The nuclei of all cells were stained with DAPI (b/ue, $n=5$. (B) Comparison of the number of TUNEL-positive cells with EXO1 or EXO2 treatment. (C) Lactate dehydrogenase (LDH) release for the detection of cell membrane pore formation $(n=5)$. (D-H) Western blot analysis of NLRP3, ASC, active caspase-1, and active GSDMD (N-terminal, $n=5$ ). Data are presented as the mean \pm SD. ${ }^{\star \star \star} P<0.001$ vs. the permanent distal middle cerebral artery occlusion (pMCAO) group. ${ }^{\prime} P<0.05,{ }^{\# \# P}<0.01,{ }^{\# \#} P<0.001$ vs. the EXO1 group. 


\section{Melatonin-Exosomes Protected Against Ischemia-Induced Pyroptosis Through an NLRP3-Mediated Pathway}

Inflammation-related pyroptosis is reported to play an important role in the secondary injury of stroke (Zhang D. et al., 2019). Studies have confirmed that pyroptosis reached its peak at 24-48 h post-stroke, then gradually decreased (Shi et al., 2017; Evavold et al., 2018). To investigate the regulatory roles of melatonin (MT)-exosomes in the pyroptotic process, we analyzed the anti-apoptosis effect after ischemic stroke. The TUNEL assay showed a noticeable decrease in apoptotic death in the EXO1 group, from approximately $69.11 \pm 5.508 \%$ to $51.5 \pm 5.679 \%$. Meanwhile, the EXO2 group exhibited a more significant suppression of cell death $(P<0.01$, compared with the EXO1 group) (Figures 3A,B), indicating that melatonin enhanced the inhibitory effect of adult rat plasma exosomes against ischemia-induced cell death. In addition, cleavage of GSDMD (p30) (Figures 3D,H) and increased $\mathrm{LDH}$ secretion (Figure 3C) after cerebral ischemia indicated GSDMD membrane pore formation (Bortolotti et al., 2018) and cellular content release. With regard to GSDMD and LDH, the EXO2 group showed a more significant reduction compared with the EXO1 group, indicating that MT-exosomes are more conducive to the prevention of pyroptotic cell death in the pMCAO model. Moreover, by detecting the NLRP3-caspase-1 axis (Figures 3D-G), the results revealed that the NLRP3 inflammasome assembled proteins, including NLRP3, ASC, and caspase-1 (p20), were all decreased after exosome administration, and the EXO2 group showed a larger inhibitory action on NLRP3 inflammasome assembly $(P<0.01$, compared with the EXO1 group). These results demonstrated that adult plasma exosomes can inhibit NLRP3-mediated pyroptosis after stroke, and the inhibitory effect could be strengthened with melatonin treatment.

\section{Melatonin-Treated Exosomes Reduced Microglia and Neuron Pyroptosis in an Ischemic Stroke Model}

It has been proven that inflammatory pyroptosis in the brain after stroke is mainly mediated by the microglia (Mamik and Power, 2017; Fricker et al., 2018). Therefore, we performed immunofluorescence staining of the brain tissue to explore microglial pyroptosis. The results revealed that the number of pyroptotic microglia in the pMCAO group was approximately $54.56 \pm 3.39$, which was significantly higher than that in the sham group and was in accordance with a previous study. After treatment with EXO1 and EXO2, the pyroptotic numbers decreased to $39.22 \pm 1.698$ and $25.89 \pm 2.137$, respectively $(P<0.001$, IBA1 stains the microglia and caspase- 1 stains pyroptotic cells) (Figure 4), which were in accordance with

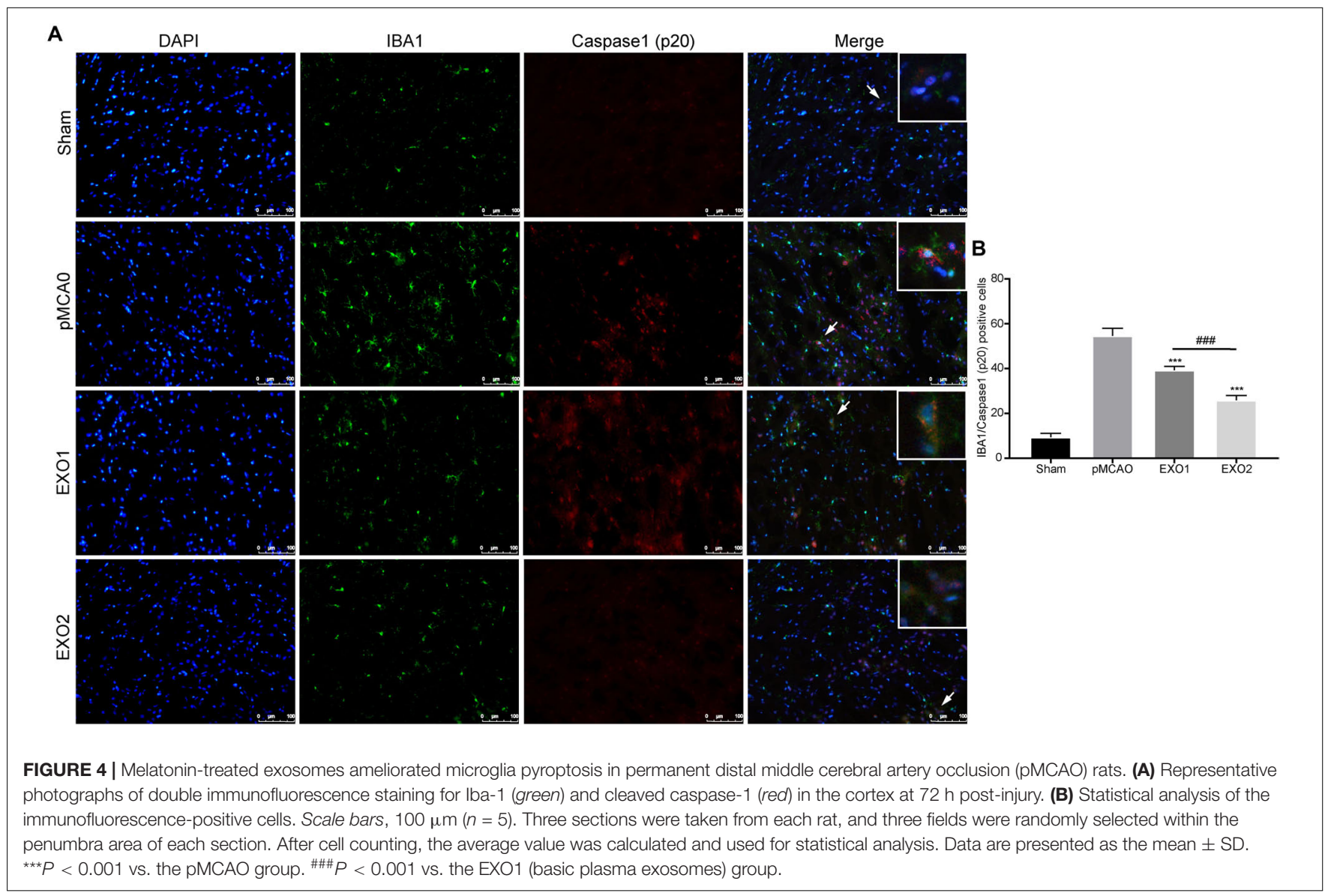


the former results. These results indicate that plasma exosomes pretreated with melatonin can better inhibit the occurrence of microglial pyroptosis, thereby blocking the initiation of the inflammatory response after stroke.

The anti-pyroptotic role in neurons was also detected. A former result (Figure 3) has declared the protective role of exosomes in preventing neuronal death in ischemic penumbra. Here, we detected the pyroptosis occurrence in neurons around the infarction area. The result showed that the number of pyroptotic neurons in the pMCAO group was $42.24 \pm 3.65$, while with treatments of EXO1 and EXO2, the pyroptotic numbers decreased to $30.64 \pm 3.00$ and $21.49 \pm 2.36$, respectively (Figure 5), indicating a more powerful protective effect of the melatonin-treated exosomes in preventing neurons from pyroptotic death.

\section{Melatonin-Treated Exosomes Alleviated the Post-stroke Inflammatory Response Through the TLR4/NF-кB Pathway}

Persistent neuroinflammation is commonly observed after ischemic stroke. To investigate the regulation of exosomes on the global inflammatory response after ischemic stroke, we examined the inflammatory and anti-inflammatory cytokine secretion profiles. Our data showed that basal plasma exosome administration effectively reduced the ischemia-induced inflammatory cytokine levels, including IL-1 $\beta$, IL-18, IL-6, TNF$\alpha$, and HMGB1, and increased the anti-inflammatory cytokines TGF- $\beta$ and IL-10 secretion, indicating its anti-inflammatory role in ischemic stroke (Figures 6A-G). MT-exosome treatment exhibited a more significant suppression of IL-1 $\beta$, IL-18, IL-6, TNF- $\alpha$, and HMGB1 secretions and increases in IL-10 and TGF$\beta$, suggesting that melatonin enhances the anti-inflammatory potential of plasma exosomes.

The alternative expressions of the downstream inflammatory factors (IL-6, TNF- $\alpha$, and HMGB1) indicated the possibility of regulating upstream regulatory elements. To investigate the regulatory mechanism of exosomes on the inflammatory response, the Toll-like receptors/nuclear factor kappa-B (TLR/NF-кB) pathway-associated proteins were detected. As shown in Figures $\mathbf{6 H}-\mathbf{L}$, the protein expressions of TLR4 and NF- $\mathrm{B}$ p 65 in the cerebral cortex increased significantly in the pMCAO group compared with those in the sham group $(P<0.01)$, while the administration of EXO1 greatly reduced the expression of NF-кB p65. In comparison with the EXO1 group, EXO2 treatment resulted in a more pronounced reduction in both TLR4 and NF- $\kappa \mathrm{B}$ expressions $(P<0.05)$, suggesting that melatonin enhances the antiinflammatory potential of plasma exosomes through the TLR4/NF- $\mathrm{B}$ pathway.

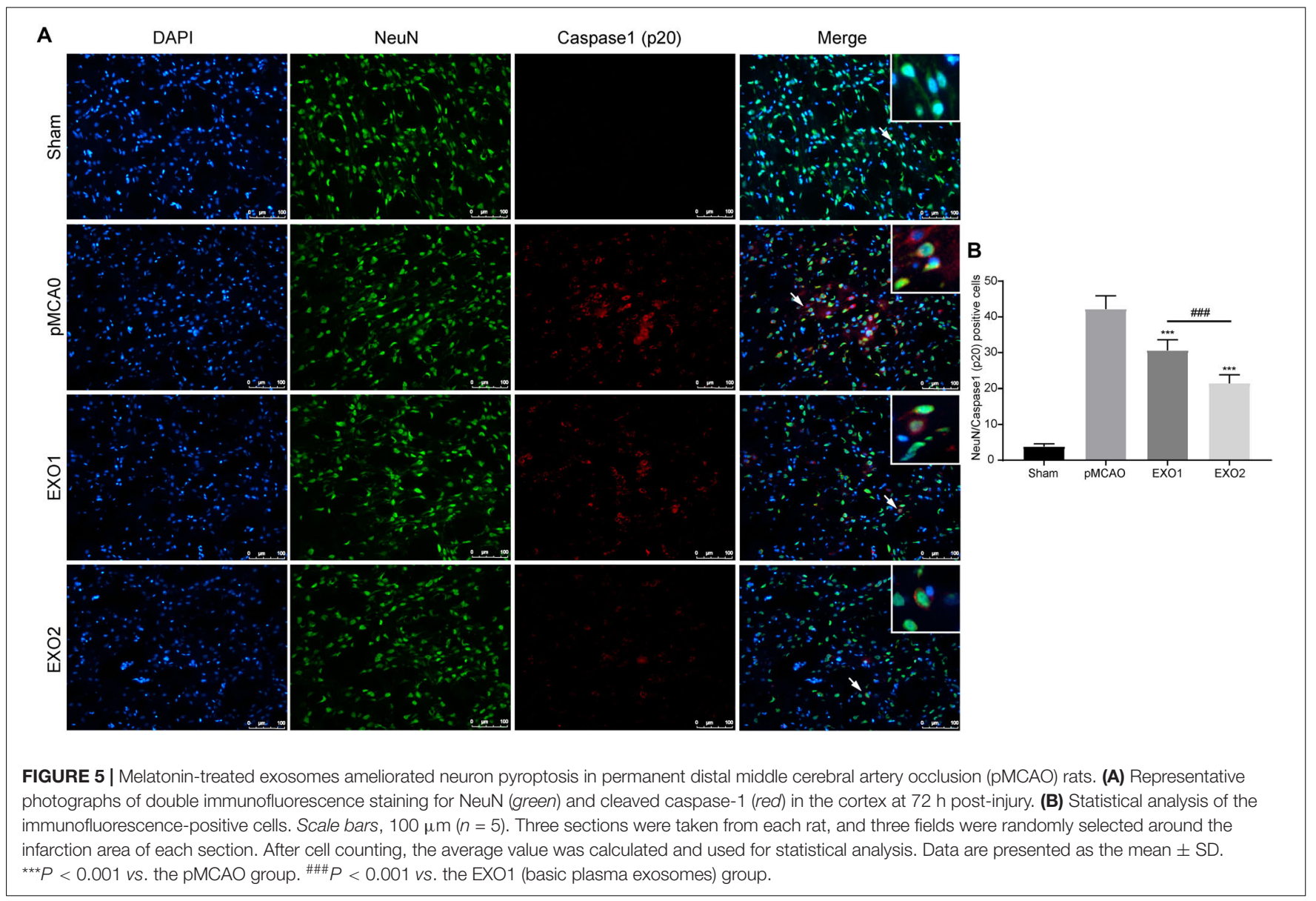


A

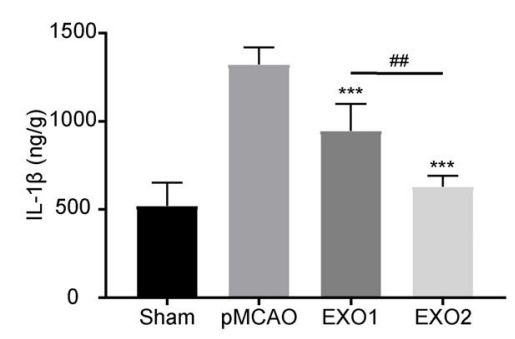

D

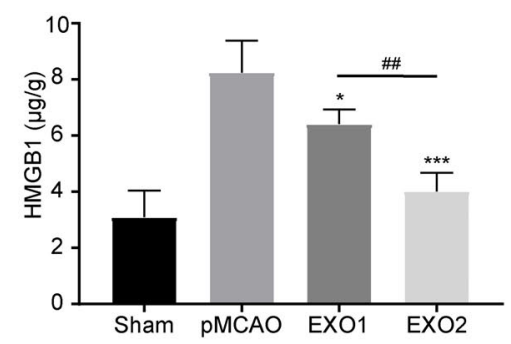

G

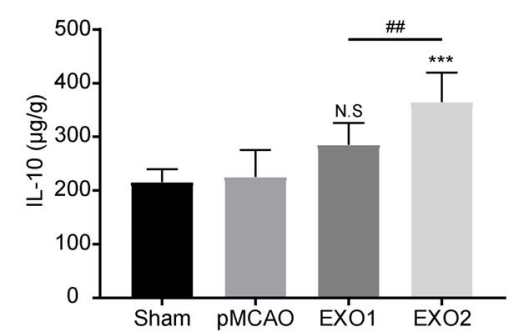

J

TLR4

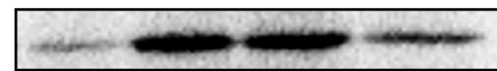

$\mathrm{NF}-\mathrm{kB}$

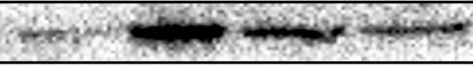

$\beta$-actin

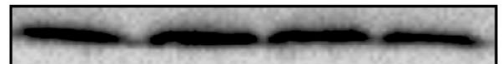

B

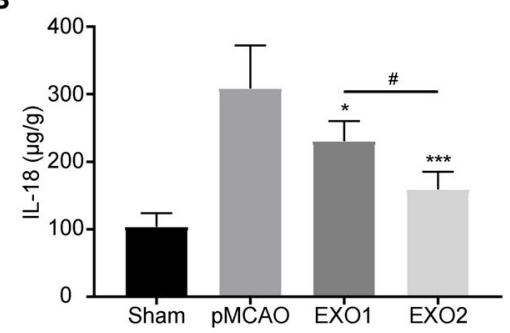

E

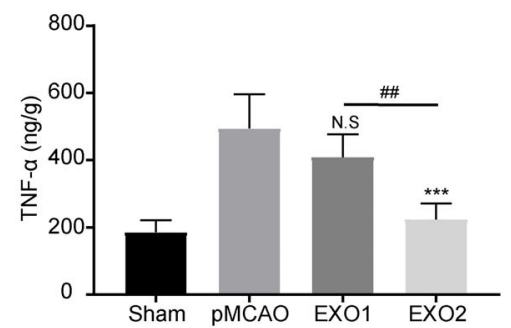

H

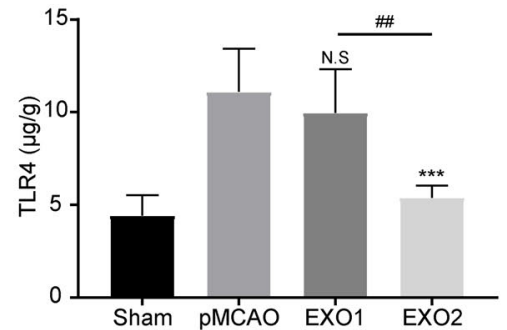

C

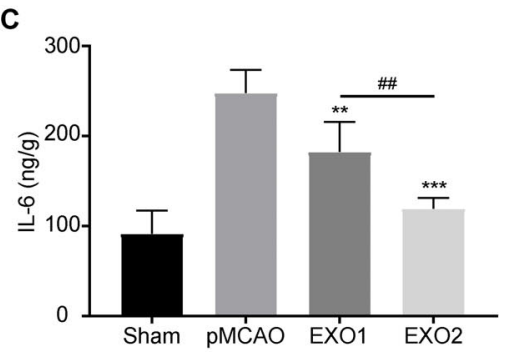

$\mathbf{F}$

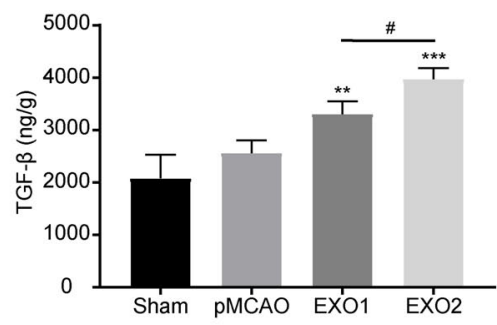

I

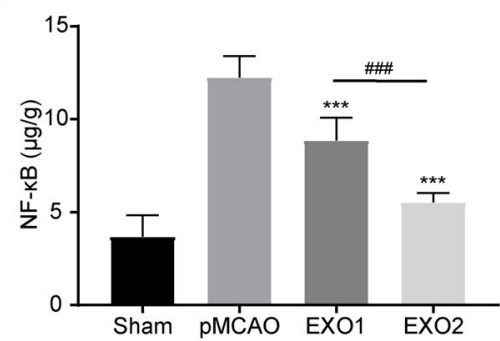

L

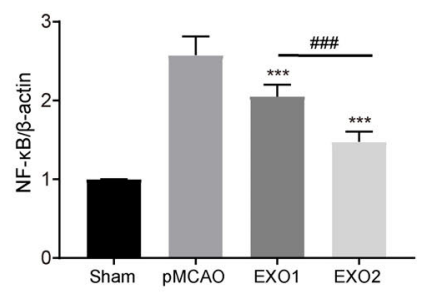

FIGURE 6 | Basic plasma exosomes (EXO1) and melatonin-treated plasma exosomes (EXO2) reduced the expression of inflammatory cytokines in the cortex after permanent distal middle cerebral artery occlusion (pMCAO) through the TLR4/NF-kB pathway. (A-G) The concentrations of the inflammatory-associated cytokines in the cortex were detected through enzyme-linked immunosorbent assay (ELISA) at $72 \mathrm{~h}(n=5)$. (H-L) The levels of TLR4 and NF-kB in the cortex were measured by ELISA and Western blotting at $72 \mathrm{~h}(n=5)$. Data are presented as the mean $\pm \mathrm{SD}$. ${ }^{\star} P<0.05,{ }^{\star \star} P<0.01,{ }^{\star \star \star} P<0.001$ vs. the pMCAO group. ${ }^{\#} P<0.05$,

${ }^{\# \#} P<0.01,{ }^{\# \#} P<0.001$ vs. the EXO1 group.

\section{miRNA Expression Profiles in Adult Rat Plasma Exosomes}

The top expressed miRNAs from the plasma exosomes were evaluated and the GO functions of the target genes were analyzed. Figures 7A,B show the enrichment score values of the top significant enrichment terms (fold_enrichment) in the EXO1 and EXO2 groups. GO function analysis revealed that the miRNA target genes were involved in biological processes, cellular components, and molecular functions, indicating a comprehensive regulatory effect of miRNAs. Compared with the basic plasma exosome, the melatonin-treated exosomes exhibited more functions involved in neurological processes, including neurogenesis, neuron differentiation, apoptosis, and angiogenesis, which was in line with the neuroprotective effect of EXO2. 


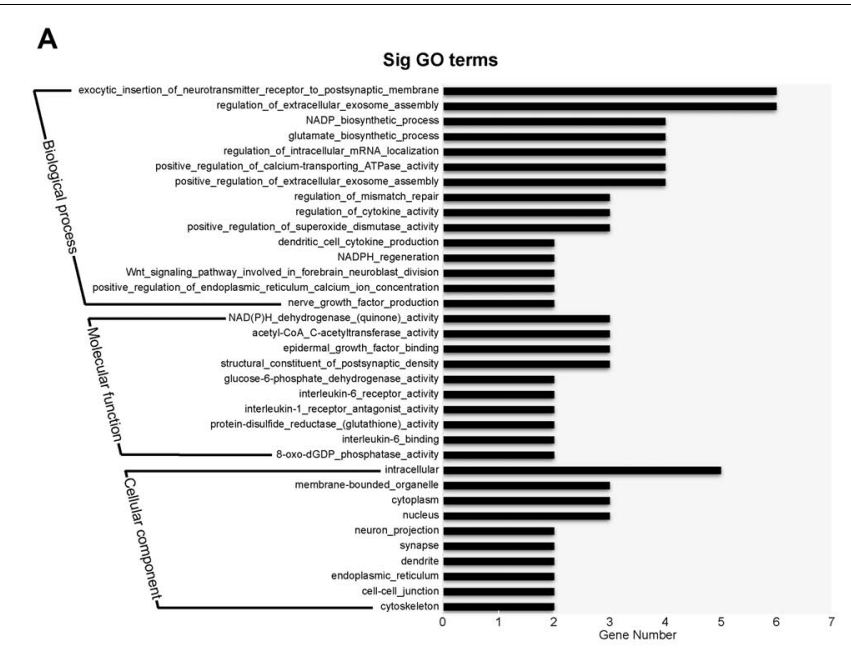

C

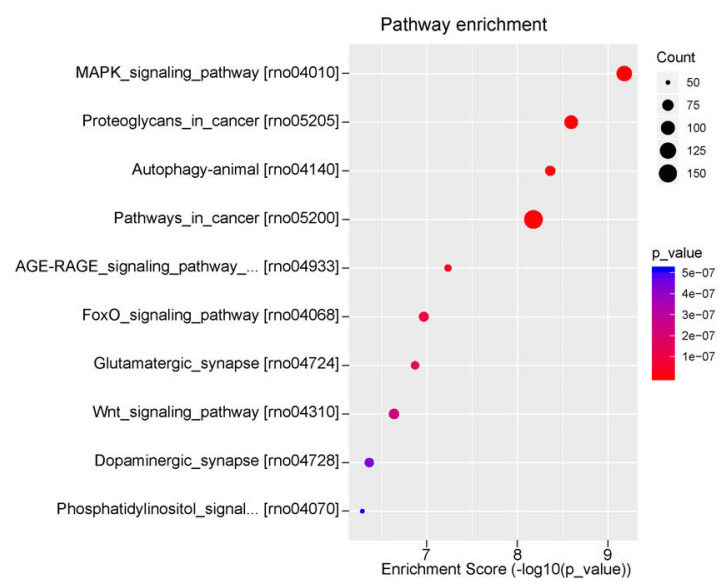

B

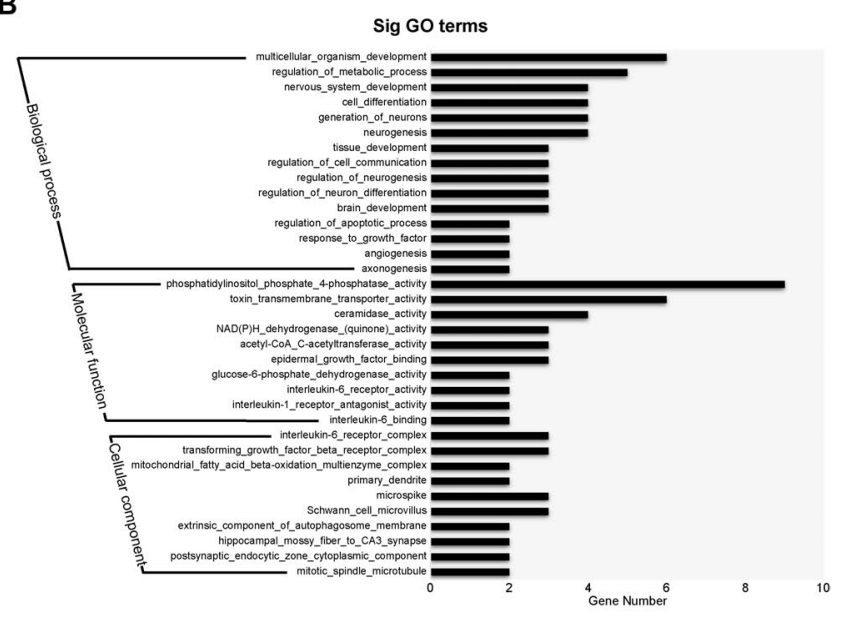

D

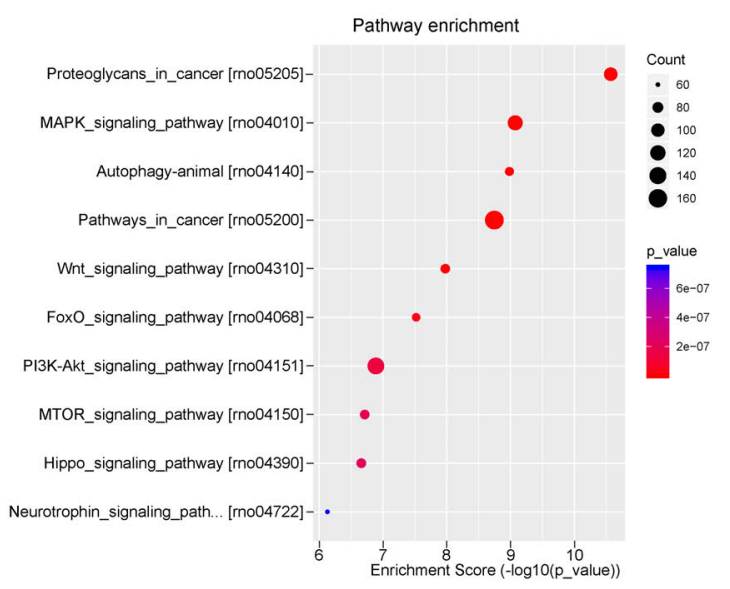

FIGURE 7 | Gene Ontology (GO) classification and GO term enrichment of the top expressed microRNAs (miRNAs) in basal plasma exosomes and melatonin-treated exosomes. (A,B) Gene Ontology classification of the potential target genes cleaved by miRNA enrichment in basal plasma exosomes (A) and melatonin-treated exosomes (B) based on their involvement in various biological processes. (C,D) Pathway enrichment of the predicted targets of the differentially expressed miRNAs in basal plasma exosomes (C) and melatonin-treated exosomes (D).

The most enriched miRNAs in EXO1 (Figure 7C) mainly participated in the MAPK signaling pathway, glutamatergic synapse, Wnt signaling pathway, dopaminergic synapse, and phosphatidylinositol signaling pathway. After melatonin treatment, the miRNA expression profiles were largely altered. Compared with the miRNAs in EXO1 (Figure 7D), the miRNA enrichment in EXO2 was more involved in the PI3K-Akt signaling pathway, MTOR signaling pathway, Hippo signaling pathway, and neurotrophin signaling pathway. These data suggest that melatonin treatment alters the miRNA composition and cellular functions, indicating its neural regulatory effects.

\section{Melatonin Alters MicroRNA Expression in Rat Plasma Exosomes}

By detecting the miRNA variation profiles in the melatonintreated plasma exosome, a total of 60 significantly differentially expressed miRNAs $\left(P_{\mathrm{FC}} \leq 0.05\right)$ were identified (after removal of repetitive miRNAs). Detailed information regarding the 12 upregulated and 47 downregulated miRNAs is summarized in Tables 1, 2. Among these differentially expressed miRNAs, a certain number of miRNAs have been reported to be involved in neurological processes, including neural apoptosis, autophagy, inflammation, and angiogenesis.

To determine the involvement of these differentially expressed miRNAs in the neural recovery process, we performed a Gene Ontology classification based on their involvement in predicted cellular pathways. A volcano plot of the altered miRNAs is presented in Figure 8. GO classification of potential target genes and their involved biological processes are analyzed in Figures 8B-E. The GO classification revealed that altered miRNAs were involved in important processes such as nerve growth factor production, Wnt signaling pathway, and transsynaptic signaling pathway (Figures 8B-E). A subsequent analysis of the modulation of these genes revealed the regulatory effect of melatonin through exosome secretion. 
TABLE 1 | The upregulated miRNAs in the plasma exosome via melatonin treatment.

\begin{tabular}{|c|c|c|c|c|}
\hline Mature_ID & $\log 2 \mathrm{FC}$ & $P$-value & $\begin{array}{l}\text { Involved } \\
\text { neurological } \\
\text { process }\end{array}$ & Resources \\
\hline$m i R-129-2-3 p$ & 267.58 & 0.038 & Autophagy & Sun et al., 2019 \\
\hline miR-496-3p & 128.13 & 0.044 & & \\
\hline miR-212-5p & 23.27 & 0.029 & $\begin{array}{l}\text { Ferroptosis; } \\
\text { neuroprotection }\end{array}$ & Xiao et al., 2019 \\
\hline$m i R-138-5 p$ & 15.97 & 0.041 & Neuroprotection & Deng et al., 2019 \\
\hline miR-501-3p & 8.17 & 0.023 & AD serum biomarker & Hara et al., 2017 \\
\hline miR-363-3p & 3.82 & 0.013 & Cognitive function & Jiang et al., 2018 \\
\hline$m i R-451-5 p$ & 3.60 & 0.017 & & \\
\hline miR-184 & 3.35 & 0.022 & $\begin{array}{l}\text { Neuroprotection; } \\
\text { inflammation; } \\
\text { anti-apoptosis }\end{array}$ & $\begin{array}{l}\text { Chen and Stallings, } \\
\text { 2007; McKiernan } \\
\text { et al., 2012; Qin } \\
\text { et al., } 2015\end{array}$ \\
\hline $\operatorname{miR}-9 a-5 p$ & 3.28 & 0.025 & $\begin{array}{l}\text { Anti-Inflammation; } \\
\text { Neuroprotection }\end{array}$ & Yuze et al., 2020 \\
\hline miR-205 & 3.06 & 0.042 & PD biomarker & $\begin{array}{l}\text { Marques et al., } \\
2017\end{array}$ \\
\hline miR-144-3p & 2.87 & 0.046 & Neuroprotection & Li et al., 2018 \\
\hline miR-92a-3p & 2.85 & 0.040 & $\begin{array}{l}\text { Post-stroke } \\
\text { depression }\end{array}$ & He et al., 2017 \\
\hline
\end{tabular}

\section{DISCUSSION}

Inflammation plays an important role in secondary injury after permanent ischemic stroke. However, the detailed underlying mechanism of the inflammation process remains unclear. Recently, emerging studies have reported that pyroptosis, a caspase-1-dependent inflammasome-mediated programmed necrosis, greatly contributes to neuronal cell death and the inflammation response. Distinct from apoptosis and other types of programmed cell death, pyroptosis is a lytic process featuring cell swelling and large bubbles emanating from the plasma membrane. In cerebral ischemia, the NLR inflammasome assembles through the recognition of PAMPs and recruitment of pro-caspase-1 with the adapter protein ASC. Upon activation, active caspase- 1 cleaves the precursor of IL-1 $\beta$ and IL-18 into their mature forms. In the meantime, cleavage of the $\mathrm{N}$-terminal domain of GSDMD forms cellular membrane GSDMD pores, which subsequently release mature IL-1 $\beta$ and IL18 into intercellular spaces and cause pyroptosis. In this study, we confirmed that intravenous injection of plasma exosomes effectively improved functional deficit recovery through the inhibition of neuronal cell pyroptosis and pyroptosis-induced inflammation. The results revealed that the administration of exosomes clearly inhibited caspase-1 activation, which subsequently prevented GSDMD cleavage and membrane GSMDM pore formation that leads to the secretion of inflammatory cytokines, including IL18, IL-1 $\beta$, TNF- $\alpha$, IL6, and HMGB1.

Previous studies have shown that coalescing young and old vessels inhibited inflammation reactions in older mice (Wang et al., 2018; Zhang and Jin, 2020), indicating that there is

TABLE 2 | The downregulated miRNAs in the plasma exosome via melatonin treatment.

\begin{tabular}{cclc}
\hline Mature_ID log2FC $P$-value & $\begin{array}{l}\text { Involved } \\
\text { neurological } \\
\text { process }\end{array}$ & Resources \\
& &
\end{tabular}

\begin{tabular}{|c|c|c|c|c|}
\hline miR-381-3p & -10.46 & 0.001 & & \\
\hline miR-295-3p & -9.88 & 0.005 & & \\
\hline miR-216b-5p & -9.76 & 0.005 & & \\
\hline miR-217-5p & -9.62 & 0.0001 & & \\
\hline miR-409a-5p & -9.37 & 0.007 & & \\
\hline$m i R-216 a-5 p$ & -9.35 & 0.010 & $\begin{array}{l}\text { Microglia } \\
\text { polarization }\end{array}$ & Liu et al., 2020 \\
\hline miR-770-3p & -8.82 & 0.015 & Aging & Lee E.K. et al., 2018 \\
\hline$m i R-466 b-3 p$ & -8.80 & 0.021 & & \\
\hline$m i R-466 c-3 p$ & -8.76 & 0.022 & & \\
\hline$m i R-493-5 p$ & -8.68 & 0.0002 & & \\
\hline miR-193b-3p & -8.38 & 0.002 & Ischemia marker & Chang and Lai, 2012 \\
\hline $\operatorname{miR}-483-5 p$ & -8.38 & 0.007 & $\begin{array}{l}\text { Angiogenesis } \\
\text { inhibitor }\end{array}$ & Qiao et al., 2011 \\
\hline miR-431 & -8.37 & 0.031 & $\begin{array}{l}\text { Hypertension and } \\
\text { vascular injury; } \\
\text { neuroprotection }\end{array}$ & $\begin{array}{l}\text { Han et al., 2018; Huo } \\
\text { et al., } 2019\end{array}$ \\
\hline miR-325-5p & -8.36 & 0.024 & & \\
\hline miR-17-2-3p & -8.32 & 0.037 & $\begin{array}{l}\text { Oxidation; } \\
\text { inflammation }\end{array}$ & Xu et al., 2018 \\
\hline $\operatorname{miR}-125 a-3 p$ & -8.19 & 0.027 & & \\
\hline$m i R-342-5 p$ & -8.11 & 0.028 & $\begin{array}{l}\text { Cell proliferation } \\
\text { and differentiation; } \\
\text { inflammation }\end{array}$ & $\begin{array}{l}\text { Wei et al., 2013; Gao } \\
\text { et al., } 2017\end{array}$ \\
\hline
\end{tabular}

miR-122-3p $\quad-8.04 \quad 0.030$

miR-881-3p $\quad-7.87 \quad 0.035$

miR-3065-5p $\quad-7.79 \quad 0.042$

$\begin{array}{lll}\text { miR-204-3p } & -7.67 & 0.038\end{array}$

miR-136-5p $\quad-7.60 \quad 0.001$

miR-130b-5p $\quad-7.57 \quad 0.012$

$\begin{array}{lll}\text { miR-485-5p } & -7.39 & 0.049\end{array}$

miR-466b-2-3p $\quad-7.12 \quad 0.019$

miR-466b-4-3p $\quad-7.12 \quad 0.019$

$\begin{array}{lll}\text { miR-30b-3p } & -7.12 \quad 0.026\end{array}$

$\begin{array}{lll}\text { miR-1b } & -6.99 & 0.0002\end{array}$

miR-877 $\quad-6.49 \quad 0.020$

miR-299a-3p $\quad-6.35 \quad 0.020$

$\begin{array}{lll}m i R-494-3 p & -6.29 & 0.011\end{array}$

$\begin{array}{lll}m i R-152-5 p & -5.81 & 0.037\end{array}$

miR-34b-5p $\quad-5.45 \quad 0.041$

miR-206-3p $\quad-5.42 \quad 0.011$

$\begin{array}{lll}m i R-497-5 p & -3.69 & 0.043\end{array}$

miR-143-5p $\quad-3.50 \quad 0.022$

$\begin{array}{lll}m i R-195-5 p & -3.17 \quad 0.014\end{array}$

$\begin{array}{lll}m i R-122-5 p & -3.14 & 0.0003\end{array}$

$\begin{array}{lll}m i R-134-5 p & -2.88 & 0.048\end{array}$

$\begin{array}{lll}m i R-145-3 p & -2.59 & 0.049\end{array}$

miR-146b-5p $\quad-2.50 \quad 0.003$

$\begin{array}{lll}\text { miR-199a-3p } & -2.45 \quad 0.005\end{array}$
Neurotoxicity Deng et al., 2020

Neuropathic pain Wen et al., 2019

BBB permeability Zhu et al., 2019

Anti-angiogenesis Sandrim et al., 2016

Neuropathic pain Z Zhu et al., 2019

Macrophage Huang et al., 2019

polarization

Anti-inflammation Chang and Lai, 2012 
TABLE 2 | Continued

\begin{tabular}{|c|c|c|c|c|}
\hline Mature_ID & $\log 2 F C$ & $P$-value & $\begin{array}{l}\text { Involved } \\
\text { neurological } \\
\text { process }\end{array}$ & Resources \\
\hline$m i R-199 a-5 p$ & -2.42 & 0.008 & $\begin{array}{l}\text { Anti-inflammation; } \\
\text { anti-apoptosis }\end{array}$ & $\begin{array}{l}\text { Li M. et al., 2019; Yu } \\
\text { et al., } 2020\end{array}$ \\
\hline $\operatorname{miR}-143-3 p$ & -2.33 & 0.006 & $\begin{array}{l}\text { Ischemia- } \\
\text { reperfusion } \\
\text { injury }\end{array}$ & Chang et al., 2019 \\
\hline miR-152-3p & -2.09 & 0.010 & $\begin{array}{l}\text { Anti-apoptosis; } \\
\text { anti-oxidation }\end{array}$ & Zhang A. et al., 2019 \\
\hline miR-100-5p & -1.95 & 0.010 & & \\
\hline $\operatorname{miR}-99 a-5 p$ & -1.64 & 0.028 & $\begin{array}{l}\text { Ischemic stroke } \\
\text { biomarker }\end{array}$ & Zhao et al., 2017 \\
\hline
\end{tabular}

BBB, blood-brain barrier.

an anti-inflammatory effect of plasma exosomes. In contrast to cell type-specific exosomes in a highly controlled process, the diversity of plasma cells determines its functional diversity. Growing evidence has shown that plasma exosomes deliver various proteins, lipids, nucleic acids, and small regulatory RNAs for cell communication and physiological regulation (Lee B.R. et al., 2018; Zhang and Jin, 2020). Zhou et al. reported that serum exosomes attenuate $\mathrm{H}_{2} \mathrm{O}_{2}$-induced apoptosis in rat $\mathrm{H} 9 \mathrm{C} 2$ cells via ERK1/2 (Li P. et al., 2019). Riquelme et al. identified that endogenous plasma exosomes can communicate TLR4 relative signals and provide protection against ischemia and reperfusion injury in the myocardium (Minghua et al., 2018). However, to date, no studies have explored the biological function of plasma on cerebral ischemia. Here, we verified that plasma exosomes inhibited ischemia-induced inflammation through NF$\kappa \mathrm{B}$ induction. Meanwhile, melatonin treatment was found to enhance its therapeutic effect on ischemic stroke through the TLR4/NF- $\mathrm{B}$ pathway. The internal molecular mechanism may lie in the specific composition of the exosomes.

To date, melatonin has demonstrated its diverse pharmacological functions against ischemic brain injury, including circadian rhythm regulation, anti-oxidation, antiinflammation, and anti-apoptosis (Sinha et al., 2001; Feng et al., 2017). Nevertheless, whether melatonin stimulus acts in a paracrine fashion to exert its neuroprotective effect requires further investigation. It has been reported that melatonin reconditioning increases the expression of matrix metalloproteinase-9 (MMP-9) and MMP-13 and decreased the expression of TGF- $\beta$ to restrain fibrosis (Saberi et al., 2019). In addition, melatonin-stimulated mesenchymal stem cell (MSC)derived exosomes enhance functional recovery in acute liver ischemia-reperfusion injury, indicating that melatonin alters the exosome content for modulation of the microenvironment by paracrine mechanisms (Yoon et al., 2020). The present study revealed that melatonin enhanced the therapeutic effect of plasma exosomes on ischemia-induced inflammation and inflammation-dependent pyroptosis through the TLR4/NF- $\mathrm{B}$ pathway, suggesting that component alteration and nerve beneficial substances are induced after melatonin administration. The downregulation of exosomal miR-100-5p and miR-199a-5p under melatonin treatment has been identified to directly

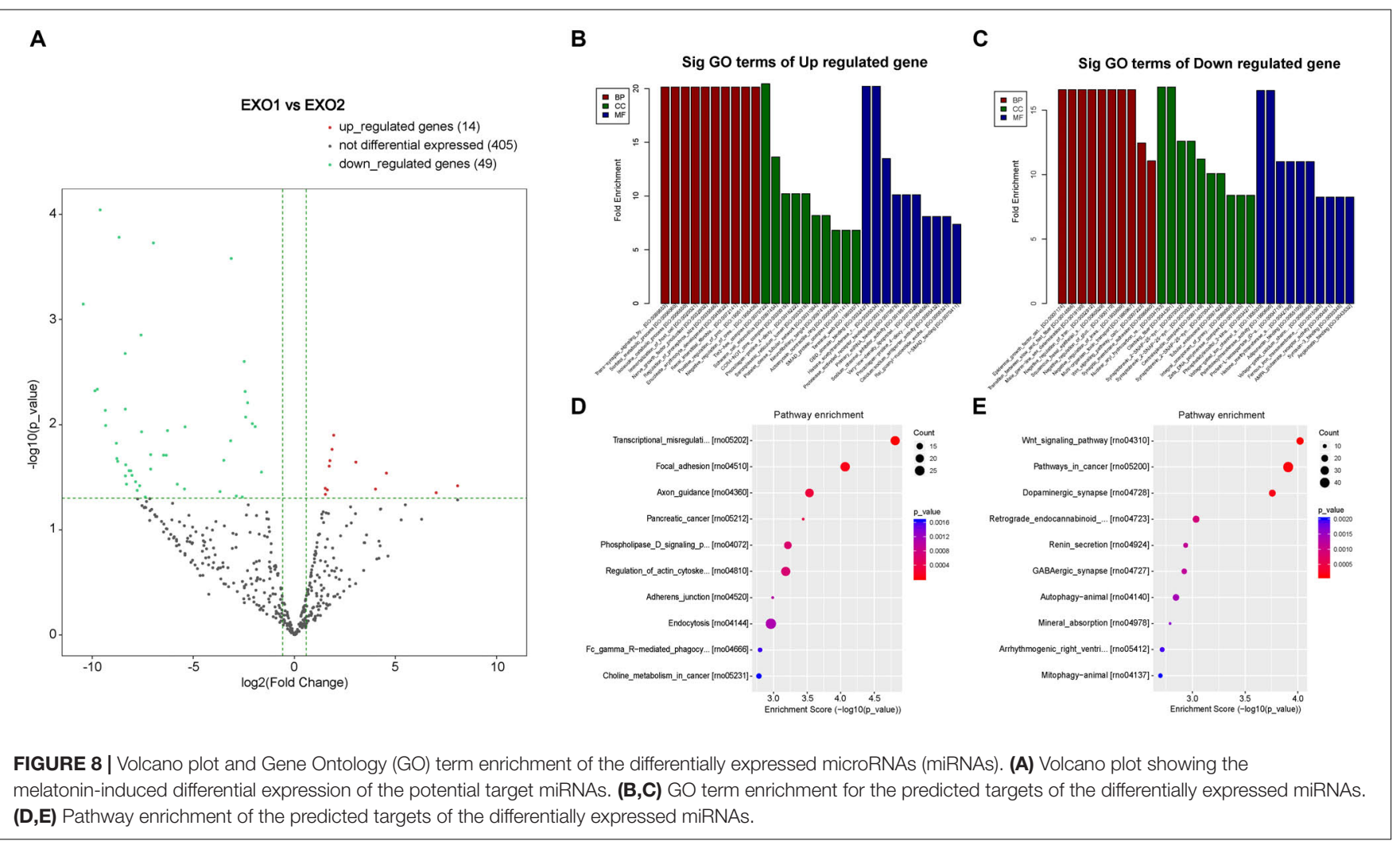


regulate TLR4, suggesting the regulatory effects of melatonintreated exosomal miRNAs. All of these results confirmed that melatonin might exert its neurological protection through the stimulation and alteration of the exosome content; nevertheless, due to the complicated composition of exosomes, we cannot exclude other mechanisms underlying its bioactivity in addition to miRNA differential expression.

As the main immune response cells in brain tissue, the status of the microglia determines the cerebral inflammation reaction. Studies have confirmed that ischemia stimulates microglial activation and promotes M1 polarization, in which the microglia secrete inflammatory mediators. With the occurrence of pyroptosis in the microglia, intracellular inflammatory mediators are largely released into the extracellular matrix, causing inflammatory injury and neuronal cell death in adjacent cells. Through an immunofluorescence assay, we confirmed the existence of pyroptosis in the microglia. The inflammatory cytokine secretion profiles demonstrated the antiinflammatory and anti-pyroptosis activity of plasma exosomes, which eventually prevented adjacent neuronal cells from undergoing pyroptosis and improved nerve function recovery.

Exosomes manipulate intercellular communication through their embedded content, including proteins and regulatory non-coding RNAs. miRNAs have been considered as key and are the most studied regulatory molecules. The exosomal miRNAs that are involved in neuronal injury and inflammation signaling may provide key insights into the mechanisms involved in ischemic injury. With systematic investigation of the top-expressed miRNA profiles, we found that exosomal miRNAs are involved in various biological processes and molecular functions, including apoptosis, dopaminergic synapse, neurotrophin signaling pathway, Wnt signaling pathway, mTOR signaling pathway, and PI3K-Akt signaling pathway, suggesting regulatory roles in the neuroprotective effect against cerebral ischemia injury.

Based on studies indicating an altered miRNA profile upon external environmental stimulus, we extended plasma exosome therapy to tailor exosomal miRNA content. Identification of the differentially expressed miRNAs in the melatonin-treated plasma exosomes provided us with new directions for the exploration of therapeutic mechanisms. On analyzing the miRNA profiles, 12 upregulated and 47 downregulated miRNAs were discovered upon melatonin treatment. Among the upregulated miRNAs (Table 1), miR-212-5p was reported to attenuate ferroptotic cell death partially by targeting Ptgs 2 in Neuro-2a cell lines (Xiao et al., 2019). The overexpression of miR-138-5p reduces neurological impairment and confers neuroprotection to astrocytes following ischemic stroke (Deng et al., 2019). miR-184 (Qin et al., 2015), miR-9a-5p (Yuze et al., 2020), and miR144-3p (Li et al., 2018) have been reported to be involved in the inflammatory response and neuronal death against ischemic stroke. Some miRNAs, not present in EXO1, were upregulated in EXO2, suggesting that melatonin stimulates their transcription. Moreover, several miRNAs were downregulated in EXO2. Among the downregulated miRNAs, miR-17-3p was found to activate the Notch $1 / N F-\kappa B$ pathways and downregulate the mitochondrial antioxidant enzymes Mn-SOD, Gpx2, and TrxR2
(Xu et al., 2018); miR-195-5p and miR-145-3p were reported to regulate macrophage polarization (Huang et al., 2019; Lin et al., 2019). Suppressing miR-152-3p protects against ischemiareperfusion injuries via the activation of the AMPK/Foxo1 pathway (Zhang A. et al., 2019). These findings support and provide insights into the molecular mechanisms underlying enhancement in neurological recovery with melatonin treatment. However, further discussion of non-conformable cases is needed. miR-152-3p and miR-199a-5p, identified as neuronal protective molecules against cerebral ischemic injury or oxygen/glucose deprivation (OGD)-induced injury, were downregulated upon melatonin treatment (Li M. et al., 2019; Zhang A. et al., 2019), whereas miR-144-3p, upregulated with melatonin treatment, was shown to increase OGD/R-induced neuronal injury via Nrf2/ARE signaling ( $\mathrm{Li}$ et al., 2018). Similarly, the downregulation of miR-146b-5p after melatonin treatment was confirmed to protect oligodendrocyte precursor cells from oxygen/glucose deprivation-induced injury by regulating Keap1/Nrf2 signaling (Li X. et al., 2019). Despite their comprehensive biological functions, further mechanisms involved in the melatoninenhanced neuroprotective effects by miRNA modulators and/or genetic regulators will be explored in our future work.

\section{CONCLUSION}

In summary, our study confirmed that basal rat plasma exosomes attenuated the ischemic-induced inflammatory response, neuronal apoptosis, and inflammasome-dependent pyroptosis after stroke in rats and that melatonin significantly enhanced the therapeutic effect by regulating the TLR4/NF- $\mathrm{B}$ pathway. Pretreatment with melatonin altered the microRNA profiles of the exosomes from rat plasma. The sequencing results identified effective miRNA components and their regulatory pathways involved in neurological recovery. However, the relationship between microRNAs and pyroptosis needs further research.

\section{DATA AVAILABILITY STATEMENT}

The sequencing data have been deposited in NCBI Gene Expression Omnibus (GEO, http://www.ncbi.nlm.nih.gov/geo/) with the accession number GSE147578.

\section{ETHICS STATEMENT}

The animal study was reviewed and approved by the Ethics Committee of Wenzhou Medical University.

\section{AUTHOR CONTRIBUTIONS}

SY designed the experiment and wrote the manuscript. QZ guided and provided the funding. KW, JR, HZ, JC, HN, and XL performed the experiment and collected the data. LH, MW, and 
ZL analyzed the data and revised the manuscript. All authors contributed to the article and approved the submitted version.

\section{FUNDING}

This study was financially supported by the Wenzhou Municipal Science Technology Bureau Project (Y20180153 and Y20190566), the National Natural Science Foundation of China (81820108011

\section{REFERENCES}

Alzahrani, F. A. (2019). Melatonin improves therapeutic potential of mesenchymal stem cells-derived exosomes against renal ischemia-reperfusion injury in rats. Am. J. Transl. Res. 11, 2887-2907.

Bortolotti, P., Faure, E., and Kipnis, E. (2018). Inflammasomes in tissue damages and immune disorders after trauma. Front. Immunol. 9:1900. doi: 10.3389/ fimmu.2018.01900

Chang, K. P., and Lai, C. S. (2012). Micro-RNA profiling as biomarkers in flap ischemia-reperfusion injury. Microsurgery 32, 642-648. doi: 10.1002/micr. 22046

Chang, L., Shi, R., Wang, X., and Bao, Y. (2019). Gypenoside A protects ischemia/reperfusion injuries by suppressing miR-143-3p level via the activation of AMPK/Foxo1 pathway. Biofactors 46, 432-440. doi: 10.1002/biof. 1601

Chen, Q., Huang, M., Wu, J., Jiang, Q., and Zheng, X. (2020). Exosomes isolated from the plasma of remote ischemic conditioning rats improved cardiac function and angiogenesis after myocardial infarction through targeting Hsp70. Aging 12, 3682-3693. doi: 10.18632/aging.102837

Chen, Y., and Stallings, R. L. (2007). Differential patterns of microRNA expression in neuroblastoma are correlated with prognosis, differentiation, and apoptosis. Cancer Res. 67, 976-983. doi: 10.1158/0008-5472.CAN-06-3667

Chen, Z., Zhang, Z., Zhang, D., Li, H., and Sun, Z. (2016). Hydrogen sulfide protects against TNF-alpha induced neuronal cell apoptosis through miR-4855p/TRADD signaling. Biochem. Biophys. Res. Commun. 478, 1304-1309. doi: 10.1016/j.bbrc.2016.08.116

Cheng, L., Liu, J., Liu, Q., Liu, Y., Fan, L., and Wang, F. (2017). Exosomes from melatonin treated hepatocellularcarcinoma cells alter the immunosupression status through STAT3 Pathway in Macrophages. Int. J. Biol. Sci. 13, 723-734. doi: $10.7150 /$ ijbs. 19642

Deng, C., Zhu, J., Yuan, J., Xiang, Y., and Dai, L. (2020). Pramipexole Inhibits MPP(+)-Induced Neurotoxicity by miR-494-3p/BDNF. Neurochem. Res. 45, 268-277. doi: 10.1007/s11064-019-02910-5

Deng, Y., Chen, D., Gao, F., Lv, H., Zhang, G., and Sun, X. (2019). Exosomes derived from microRNA-138-5p-overexpressing bone marrowderived mesenchymal stem cells confer neuroprotection to astrocytes following ischemic stroke via inhibition of LCN2. J. Biol. Eng. 13:71. doi: 10.1186/s13036019-0193-0

Ding, S., Liu, D., Wang, L., Wang, G., and Zhu, Y. (2020). Inhibiting MicroRNA29a protects myocardial ischemia-reperfusion injury by targeting SIRT1 and suppressing oxidative stress and NLRP3-Mediated pyroptosis pathway. J. Pharmacol. Exp. Ther. 372, 128-135. doi: 10.1124/jpet.119.256982

Eirin, A., Riester, S. M., Zhu, X. Y., Tang, H., Evans, J. M., and O’Brien, D. (2014). MicroRNA and mRNA cargo of extracellular vesicles from porcine adipose tissue-derived mesenchymal stem cells. Gene 551, 55-64. doi: 10.1016/j.gene. 2014.08.041

Evavold, C. L., Ruan, J., Tan, Y., Xia, S., Wu, H., and Kagan, J. C. (2018). The poreforming protein Gasdermin D regulates Interleukin-1 secretion from living macrophages. Immunity 48, 35.e6-44.e6. doi: 10.1016/j.immuni.2017.11.013

Feigin, V. L., Forouzanfar, M. H., Krishnamurthi, R., Mensah, G. A., Connor, M., and Bennett, D. A. (2014). Global and regional burden of stroke during 1990-2010: findings from the global burden of disease study 2010. Lancet 383, 245-254. doi: 10.1016/s0140-6736(13)61953-4

Feng, D., Wang, B., Wang, L., Abraham, N., and Tao, K. (2017). Preischemia melatonin treatment alleviated acute neuronal injury after ischemic stroke by and 81771262) and the Natural Science Foundation of Zhejiang Province (2017C03027).

\section{ACKNOWLEDGMENTS}

We would like to thank Editage ${ }^{2}$ for English language editing.

${ }^{2}$ www.editage.com.

inhibiting endoplasmic reticulum stress-dependent autophagy via PERK and IRE1 signalings. J. Pineal Res. 62:e12395. doi: 10.1111/jpi.12395

Fricker, M., Tolkovsky, A. M., Borutaite, V., Coleman, M., and Brown, G. C. (2018). Neuronal cell death. Physiol. Rev. 98, 813-880. doi: 10.1152/physrev.00011. 2017

Gao, F., Zhang, Y. F., Zhang, Z. P., Fu, L. A., Cao, X. L., and Zhang, Y. Z. (2017). miR-342-5p regulates neural stem cell proliferation and differentiation downstream to notch signaling in mice. Stem Cell Rep. 8, 1032-1045. doi: 10.1016/j.stemcr.2017.02.017

Han, X. R., Wen, X., Wang, Y. J., Wang, S., Shen, M., and Zhang, Z. F. (2018). Protective effects of microRNA-431 against cerebral ischemia-reperfusion injury in rats by targeting the Rho/Rho-kinase signaling pathway. J. Cell Physiol. 233, 5895-5907. doi: 10.1002/jcp.26394

Hara, N., Kikuchi, M., Miyashita, A., Hatsuta, H., Saito, Y., and Kasuga, K. (2017). Serum microRNA miR-501-3p as a potential biomarker related to the progression of Alzheimer's disease. Acta Neuropathol. Commun. 5:10. doi: 10. 1186/s40478-017-0414-z

He, J. R., Zhang, Y., Lu, W. J., Liang, H. B., Tu, X. Q., and Ma, F. Y. (2017). Agerelated frontal periventricular white matter hyperintensities and miR-92a-3p Are associated with early-onset post-stroke depression. Front. Aging Neurosci. 9:328. doi: 10.3389 /fnagi.2017.00328

He, Y., Hara, H., and Nunez, G. (2016). Mechanism and regulation of NLRP3 inflammasome activation. Trends Biochem. Sci. 41, 1012-1021. doi: 10.1016/j. tibs.2016.09.002

Huang, Y., Du, K. L., Guo, P. Y., Zhao, R. M., Wang, B., and Zhao, X. L. (2019). IL-16 regulates macrophage polarization as a target gene of mir-145-3p. Mol. Immunol. 107, 1-9. doi: 10.1016/j.molimm.2018.12.027

Hunsaker, M., Barba, G., Kingsley, K., and Howard, K. M. (2019). Differential MicroRNA expression of miR-21 and miR-155 within oral cancer extracellular vesicles in response to melatonin. Dent. J. 7:48. doi: 10.3390/dj7020048

Huo, K. G., Richer, C., Berillo, O., Mahjoub, N., Fraulob-Aquino, J. C., and Barhoumi, T. (2019). miR-431-5p knockdown protects against angiotensin IIinduced hypertension and vascular injury. Hypertension 73, 1007-1017. doi: 10.1161/HYPERTENSIONAHA.119.12619

Ishrat, T., Mohamed, I. N., Pillai, B., Soliman, S., Fouda, A. Y., and Ergul, A. (2015). Thioredoxin-interacting protein: a novel target for neuroprotection in experimental thromboembolic stroke in mice. Mol. Neurobiol. 51, 766-778. doi: $10.1007 / \mathrm{s} 12035-014-8766-\mathrm{x}$

Jeyabal, P., Thandavarayan, R. A., Joladarashi, D., Suresh Babu, S., Krishnamurthy, S., and Bhimaraj, A. (2016). MicroRNA-9 inhibits hyperglycemia-induced pyroptosis in human ventricular cardiomyocytes by targeting ELAVL1. Biochem. Biophys. Res. Commun. 471, 423-429. doi: 10.1016/j.bbrc.2016.02.065

Jiang, C., Logan, S., Yan, Y., Inagaki, Y., Arzua, T., and Ma, P. (2018). Signaling network between the dysregulated expression of microRNAs and mRNAs in propofol-induced developmental neurotoxicity in mice. Sci .Rep. 8:14172. doi: 10.1038/s41598-018-32474-3

Jin, R., Yang, G., and Li, G. (2010). Inflammatory mechanisms in ischemic stroke: role of inflammatory cells. J. Leukoc Biol. 87, 779-789. doi: 10.1189/jlb.1109766

Lee, B. R., Kim, J. H., Choi, E. S., Cho, J. H., and Kim, E. (2018). Effect of young exosomes injected in aged mice. Int. J. Nanomedicine 13, 5335-5345. doi: 10.2147/IJN.S170680

Lee, E. K., Jeong, H. O., Bang, E. J., Kim, C. H., Mun, J. Y., and Noh, S. (2018). The involvement of serum exosomal miR-500-3p and miR-770-3p in aging: modulation by calorie restriction. Oncotarget 9, 5578-5587. doi: 10.18632/ oncotarget.23651 
Li, M., Luan, L., Liu, Q., Liu, Y., Lan, X., and Li, Z. (2019). MiRNA-199a-5p protects against cerebral ischemic injury by down-regulating DDR1 in Rats. World Neurosurg. 131, e486-e494. doi: 10.1016/j.wneu.2019.07.203

Li, P., Liu, Z., Xie, Y., Gu, H., Dai, Q., and Yao, J. (2019). serum exosomes attenuate $\mathrm{H} 2 \mathrm{O} 2$-induced apoptosis in rat $\mathrm{H} 9 \mathrm{C} 2$ cardiomyocytes via ERK1/2. J. Cardiovasc. Transl. Res. 12, 37-44. doi: 10.1007/s12265-018-9791-3

Li, X., Zhang, W., Xiao, M., Wang, F., Zhou, P., and Yang, J. (2019). MicroRNA146b-5p protects oligodendrocyte precursor cells from oxygen/glucose deprivation-induced injury through regulating Keap1/Nrf2 signaling via targeting bromodomain-containing protein 4. Biochem. Biophys. Res. Commun. 513, 875-882. doi: 10.1016/j.bbrc.2019.04.045

Li, X., Du, N., Zhang, Q., Li, J., Chen, X., and Liu, X. (2014). MicroRNA-30d regulates cardiomyocyte pyroptosis by directly targeting foxo3a in diabetic cardiomyopathy. Cell Death Dis. 5:e1479. doi: 10.1038/cddis.2014.430

Li, Y., Zhao, Y., Cheng, M., Qiao, Y., Wang, Y., and Xiong, W. (2018). Suppression of microRNA-144-3p attenuates oxygen-glucose deprivation/reoxygenationinduced neuronal injury by promoting Brg1/Nrf2/ARE signaling. J. Biochem. Mol. Toxicol. 32:e22044. doi: 10.1002/jbt.22044

Liang, H., and Liu, Y. (2016). Gasdermins pore cell membrane to pyroptosis. Sci. China Life Sci. 59, 1090-1092. doi: 10.1007/s11427-016-0243-4

Lin, X., Wang, S., Sun, M., Zhang, C., Wei, C., and Yang, C. (2019). miR-1955p/NOTCH2-mediated EMT modulates IL-4 secretion in colorectal cancer to affect M2-like TAM polarization. J. Hematol. Oncol. 12:20. doi: 10.1186/s13045019-0708-7

Liu, W., Rong, Y., Wang, J., Zhou, Z., Ge, X., and Ji, C. (2020). Exosomeshuttled miR-216a-5p from hypoxic preconditioned mesenchymal stem cells repair traumatic spinal cord injury by shifting microglial M1/M2 polarization. J. Neuroinflammation 17:47. doi: 10.1186/s12974-020-1726-7

Liu, X., Zhang, Z., Ruan, J., Pan, Y., Magupalli, V. G., and Wu, H. (2016). Inflammasome-activated gasdermin $\mathrm{D}$ causes pyroptosis by forming membrane pores. Nature 535, 153-158. doi: 10.1038/nature18629

Longa, E. Z., Weinstein, P. R., Carlson, S., and Cummins, R. (1989). Reversible middle cerebral artery occlusion without craniectomy in rats. Stroke 20, 84-91. doi: $10.1161 / 01 . s t r .20 .1 .84$

Lotvall, J., Hill, A. F., Hochberg, F., Buzas, E. I., Di Vizio, D., and Gardiner, C. (2014). Minimal experimental requirements for definition of extracellular vesicles and their functions: a position statement from the International Society for extracellular vesicles. J. Extracell Vesicles 3:26913. doi: 10.3402/jev.v3.26913

Lugli, G., Cohen, A. M., Bennett, D. A., Shah, R. C., Fields, C. J., and Hernandez, A. G. (2015). Plasma exosomal miRNAs in persons with and without Alzheimer disease: altered expression and prospects for biomarkers. PLoS One 10:e0139233. doi: 10.1371/journal.pone.0139233

Mamik, M. K., and Power, C. (2017). Inflammasomes in neurological diseases: emerging pathogenic and therapeutic concepts. Brain 140, 2273-2285. doi: 10.1093/brain/awx133

Marques, T. M., Kuiperij, H. B., Bruinsma, I. B., van Rumund, A., Aerts, M. B., and Esselink, R. A. J. (2017). MicroRNAs in cerebrospinal fluid as potential biomarkers for Parkinson's Disease and multiple system atrophy. Mol. Neurobiol. 54, 7736-7745. doi: 10.1007/s12035-016-0253-0

McKiernan, R. C., Jimenez-Mateos, E. M., Sano, T., Bray, I., Stallings, R. L., and Simon, R. P. (2012). Expression profiling the microRNA response to epileptic preconditioning identifies miR-184 as a modulator of seizure-induced neuronal death. Exp. Neurol. 237, 346-354. doi: 10.1016/j.expneurol.2012.06.029

Minghua, W., Zhijian, G., Chahua, H., Qiang, L., Minxuan, X., and Luqiao, W. (2018). Plasma exosomes induced by remote ischaemic preconditioning attenuate myocardial ischaemia/reperfusion injury by transferring miR-24. Cell Death Dis. 9:320. doi: 10.1038/s41419-018-0274-x

Qiao, Y., Ma, N., Wang, X., Hui, Y., Li, F., and Xiang, Y. (2011). MiR-483-5p controls angiogenesis in vitro and targets serum response factor. FEBS Lett. 585, 3095-3100. doi: 10.1016/j.febslet.2011.08.039

Qin, C. Z., Lou, X. Y., Lv, Q. L., Cheng, L., Wu, N. Y., and Hu, L. (2015). MicroRNA184 acts as a potential diagnostic and prognostic marker in epithelial ovarian cancer and regulates cell proliferation, apoptosis and inflammation. Pharmazie $70,668-673$.

Rancan, L., Paredes, S. D., Garcia, C., Gonzalez, P., Rodriguez-Bobada, C., and Calvo-Soto, M. (2018). Comparison of the effect of melatonin treatment before and after brain ischemic injury in the inflammatory and apoptotic response in aged rats. Int. J. Mol. Sci. 19:2097. doi: 10.3390/ijms19072097
Ren, C., Yao, Y., Han, R., Huang, Q., Li, H., and Wang, B. (2018). Cerebral ischemia induces angiogenesis in the peri-infarct regions via Notch1 signaling activation. Exp. Neurol. 304, 30-40. doi: 10.1016/j.expneurol.2018.02.013

Saberi, K., Pasbakhsh, P., Omidi, A., Borhani-Haghighi, M., Nekoonam, S., and Omidi, N. (2019). Melatonin preconditioning of bone marrow-derived mesenchymal stem cells promotes their engraftment and improves renal regeneration in a rat model of chronic kidney disease. J. Mol. Histol. 50, 129-140. doi: 10.1007/s10735-019-09812-4

Sandrim, V. C., Dias, M. C., Bovolato, A. L., Tanus-Santos, J. E., Deffune, E., and Cavalli, R. C. (2016). Plasma from pre-eclamptic patients induces the expression of the anti-angiogenic miR-195-5p in endothelial cells. J. Cell Mol. Med. 20, 1198-1200. doi: $10.1111 /$ jcmm. 12767

Sarhan, M., Land, W. G., Tonnus, W., Hugo, C. P., and Linkermann, A. (2018). Origin and consequences of necroinflammation. Physiol. Rev. 98, 727-780. doi: 10.1152/physrev.00041.2016

Shi, J., Gao, W., and Shao, F. (2017). Pyroptosis: gasdermin-mediated programmed necrotic cell death. Trends Biochem. Sci. 42, 245-254. doi: 10.1016/j.tibs.2016. 10.004

Sinha, K., Degaonkar, M. N., Jagannathan, N. R., and Gupta, Y. K. (2001). Effect of melatonin on ischemia reperfusion injury induced by middle cerebral artery occlusion in rats. Eur. J. Pharmacol. 428, 185-192. doi: 10.1016/s0014-2999(01) 01253-5

Sun, W., Yi, Y., Xia, G., Zhao, Y., Yu, Y., and Li, L. (2019). Nrf2-miR-1293p-mTOR axis controls an miRNA regulatory network involved in HDACiinduced autophagy. Mol. Ther. 27, 1039-1050. doi: 10.1016/j.ymthe.2019. 02.010

Vanni, I., Alama, A., Grossi, F., Dal Bello, M. G., and Coco, S. (2017). Exosomes: a new horizon in lung cancer. Drug. Discov. Today 22, 927-936. doi: 10.1016/j. drudis.2017.03.004

Vicencio, J. M., Yellon, D. M., Sivaraman, V., Das, D., Boi-Doku, C., and Arjun, S. (2015). Plasma exosomes protect the myocardium from ischemiareperfusion injury. J. Am. Coll Cardiol. 65, 1525-1536. doi: 10.1016/j.jacc.2015. 02.026

Wang, W., Wang, L., Ruan, L., Oh, J., Dong, X., and Zhuge, Q. (2018). Extracellular vesicles extracted from young donor serum attenuate inflammaging via partially rejuvenating aged T-cell immunotolerance. FASEB J. 32:fj201800059R. doi: 10. 1096/fj.201800059R

Wei, Y., Nazari-Jahantigh, M., Chan, L., Zhu, M., Heyll, K., and CorbalanCampos, J. (2013). The microRNA-342-5p fosters inflammatory macrophage activation through an Akt1- and microRNA-155dependent pathway during atherosclerosis. Circulation 127, 1609-1619. doi: 10.1161/CIRCULATIONAHA.112.000736

Wen, J., He, T., Qi, F., and Chen, H. (2019). MiR-206-3p alleviates chronic constriction injury-induced neuropathic pain through targeting HDAC4. Exp. Anim. 68, 213-220. doi: 10.1538/expanim.18-0091

Wu, H., Huang, T., Ying, L., Han, C., Li, D., and Xu, Y. (2016). MiR-155 is involved in renal ischemia-reperfusion injury via direct targeting of foxo3a and regulating renal tubular cell pyroptosis. Cell Physiol. Biochem. 40, 1692-1705. doi: 10.1159/000453218

Xiao, X., Jiang, Y., Liang, W., Wang, Y., Cao, S., and Yan, H. (2019). miR-212-5p attenuates ferroptotic neuronal death after traumatic brain injury by targeting Ptgs2. Mol. Brain 12: 78. doi: 10.1186/s13041-019-0501-0

Xu, Z., Zhang, Y., Ding, J., Hu, W., Tan, C., and Wang, M. (2018). miR17-3p downregulates mitochondrial antioxidant enzymes and enhances the radiosensitivity of prostate cancer cells. Mol. Ther. Nucleic Acids 13, 64-77. doi: 10.1016/j.omtn.2018.08.009

Yoon, Y. M., Lee, J. H., Song, K. H., Noh, H., and Lee, S. H. (2020). Melatoninstimulated exosomes enhance the regenerative potential of chronic kidney disease-derived mesenchymal stem/stromal cells via cellular prion proteins. J. Pineal Res. 68:e12632. doi: 10.1111/jpi.12632

Yu, Y., Zhou, H., Xiong, Y., and Liu, J. (2020). Exosomal miR-199a-5p derived from endothelial cells attenuates apoptosis and inflammation in neural cells by inhibiting endoplasmic reticulum stress. Brain Res. 1726:146515. doi: 10.1016/j. brainres.2019.146515

Yuze, C., Huixue, Z., Xiaoyu, L., Jianjian, W., Xiaoming, Z., and Shengnan, S. (2020). Overexpression of MicroRNA-9a-5p Ameliorates NLRP1 Inflammasome-mediated Ischemic Injury in Rats Following Ischemic Stroke. Neuroscience (in press). doi: 10.1016/j.neuroscience.2020.01.008 
Zhang, A., Qian, Y., and Qian, J. (2019). MicroRNA-152-3p protects neurons from oxygen-glucose-deprivation/reoxygenation-induced injury through upregulation of Nrf2/ARE antioxidant signaling by targeting PSD-93. Biochem. Biophys. Res. Commun. 517, 69-76. doi: 10.1016/j.bbrc.2019.07.012

Zhang, D., Qian, J., Zhang, P., Li, H., Shen, H., and Li, X. (2019). Gasdermin D serves as a key executioner of pyroptosis in experimental cerebral ischemia and reperfusion model both in vivo and in vitro. J. Neurosci. Res. 97, 645-660. doi: 10.1002/jnr.24385

Zhang, X., Sai, B., Wang, F., Wang, L., Wang, Y., and Zheng, L. (2019). Hypoxic BMSC-derived exosomal miRNAs promote metastasis of lung cancer cells via STAT3-induced EMT. Mol. Cancer 18:40. doi: 10.1186/s12943-019-0959-5

Zhang, H., and Jin, K. (2020). Peripheral circulating exosomal miRNAs potentially contribute to the regulation of molecular signaling networks in aging. Int. J. Mol. Sci. 21:1908. doi: 10.3390/ijms21061908

Zhang, J., Li, S., Li, L., Li, M., Guo, C., and Yao, J. (2015). Exosome and exosomal microRNA: trafficking, sorting, and function. Genomics Proteomics Bioinformatics 13, 17-24. doi: 10.1016/j.gpb.2015.02.001

Zhao, H., Li, G., Ma, Q., Tao, Z., Wang, R., and Fan, Z. (2017). MicroRNA-99a-5p in circulating immune cells as a potential biomarker for the early diagnosis of ischemic stroke. Brain Circ. 3, 21-28. doi: 10.4103/bc.bc_1_17
Zhong, Y., Yu, C., and Qin, W. (2019). LncRNA SNHG14 promotes inflammatory response induced by cerebral ischemia/reperfusion injury through regulating miR-136-5p /ROCK1. Cancer Gene Ther. 26, 234-247. doi: 10.1038/s41417018-0067-5

Zhu, L., Lin, M., Ma, J., Liu, W., Gao, L., and Wei, S. (2019). The role of LINC00094/miR-224-5p (miR-497-5p)/Endophilin-1 axis in Memantine mediated protective effects on blood-brain barrier in AD microenvironment. J. Cell Mol. Med. 23, 3280-3292. doi: 10.1111/jcmm.14214

Conflict of Interest: The authors declare that the research was conducted in the absence of any commercial or financial relationships that could be construed as a potential conflict of interest.

Copyright (c) 2020 Wang, Ru, Zhang, Chen, Lin, Lin, Wen, Huang, Ni, Zhuge and Yang. This is an open-access article distributed under the terms of the Creative Commons Attribution License (CC BY). The use, distribution or reproduction in other forums is permitted, provided the original author(s) and the copyright owner(s) are credited and that the original publication in this journal is cited, in accordance with accepted academic practice. No use, distribution or reproduction is permitted which does not comply with these terms. 